\title{
The Camera of the MASCOT Asteroid Lander on Board Hayabusa 2
}

\author{
R. Jaumann ${ }^{1,2}$ • N. Schmitz ${ }^{1}$ A. Koncz ${ }^{1}$ - H. Michaelis ${ }^{1}$ - S.E. Schroeder ${ }^{1}$ - S. Mottola ${ }^{1}$

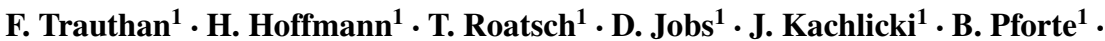 \\ R. Terzer ${ }^{1}$ - M. Tschentscher ${ }^{1}$. S. Weisse ${ }^{1}$. U. Mueller ${ }^{1}$ - L. Perez-Prieto ${ }^{3}$. B. Broll ${ }^{3}$. \\ A. Kruselburger ${ }^{3}$. T.-M. Ho ${ }^{4}$. J. Biele ${ }^{5}$ - S. Ulamec ${ }^{5}$ - C. Krause ${ }^{5}$ - M. Grott ${ }^{1}$. \\ J.-P. Bibring ${ }^{6} \cdot$ S. Watanabe ${ }^{7}$-S. Sugita ${ }^{8}$ T. Okada ${ }^{9} \cdot$ M. Yoshikawa ${ }^{9} \cdot$ H. Yabuta ${ }^{10}$
}

Received: 28 August 2015 / Accepted: 2 June 2016

(C) Springer Science+Business Media Dordrecht 2016

\begin{abstract}
The MASCOT Camera (MasCam) is part of the Mobile Asteroid Surface Scout (MASCOT) lander's science payload. MASCOT has been launched to asteroid (162173) Ryugu onboard JAXA's Hayabusa 2 asteroid sample return mission on Dec 3rd, 2014. It is scheduled to arrive at Ryugu in 2018, and return samples to Earth by 2020. MasCam was designed and built by DLR's Institute of Planetary Research, together with Airbus-DS Germany. The scientific goals of the MasCam investigation are to provide ground truth for the orbiter's remote sensing observations, provide context for measurements by the other lander instruments (radiometer, spectrometer and magnetometer), the orbiter sampling experiment, and characterize the geological context, compositional variations and physical properties of the surface (e.g. rock and regolith particle size distributions). During daytime, clear filter images will be acquired. During night, illumination of the dark surface is performed by an LED array, equipped with $4 \times 36$ monochromatic light-emitting diodes (LEDs) working in four spectral bands. Color imaging will allow the identification of spectrally distinct surface units. Continued imaging during the surface mission phase and the acquisition of image series at different sun angles over the course of an asteroid day will contribute to the physical characterization of the surface and also allow the investigation of time-dependent
\end{abstract}

\footnotetext{
R. Jaumann

ralf.jaumann@dlr.de

DLR, Institute of Planetary Research, Berlin, Germany

2 Freie Univ. Berlin, Inst. of Geosciences, Berlin, Germany

3 Airbus DS, Munich, Germany

4 DLR, Institute of Space Systems, Bremen, Germany

5 DLR-MUSC, Linder Höhe, Cologne, Germany

6 Univ. de Paris Sud-Orsay, IAS, Orsay, France

7 Dep. of Earth and Planetary Sciences, Nagoya Univ. Furo-cho Chikusa-ku, Nagoya, Japan

8 Dept. of Earth and Planetary Science, University of Tokyo, Tokyo, Japan

9 JSPEC/JAXA, Yoshinodai, Chuo, Sagamihara, Kanagawa, Japan

10 Dept. of Earth and Space Science, Osaka University, Osaka, Japan
} 
processes and to determine the photometric properties of the regolith. The MasCam observations, combined with the MASCOT hyperspectral microscope (MMEGA) and radiometer (MARA) thermal observations, will cover a wide range of observational scales and serve as a strong tie point between Hayabusa 2's remote-sensing scales $\left(10^{3}-10^{-3} \mathrm{~m}\right)$ and sample scales $\left(10^{-3}-10^{-6} \mathrm{~m}\right)$. The descent sequence and the close-up images will reveal the surface features over a broad range of scales, allowing an assessment of the surface's diversity and close the gap between the orbital observations and those made by the in-situ measurements. The MasCam is mounted inside the lander slightly tilted, such that the center of its $54.8^{\circ}$ square field-of-view is directed towards the surface at an angle of $22^{\circ}$ with respect to the surface plane. This is to ensure that both the surface close to the lander and the horizon are observable. The camera optics is designed according to the Scheimpflug principle, thus that the entire scene along the camera's depth of field (150 $\mathrm{mm}$ to infinity) is in focus. The camera utilizes a $1024 \times 1024$ pixel CMOS sensor sensitive in the 400-1000 nm wavelength range, peaking at $600-700 \mathrm{~nm}$. Together with the $\mathrm{f}-16$ optics, this yields a nominal ground resolution of $150 \mathrm{micron} / \mathrm{px}$ at $150 \mathrm{~mm}$ distance (diffraction limited). The camera flight model has undergone standard radiometric and geometric calibration both at the component and system (lander) level. MasCam relies on the use of wavelet compression to maximize data return within stringent mission downlink limits. All calibration and flight data products will be generated and archived in the Planetary Data System in PDS image format.

Keywords Hayabusa 2 · Mascot · Camera · Asteroid (162173) Ryugu · In-situ science

\section{Introduction}

MASCOT, a Mobile Asteroid Surface Scout (Ho et al. 2016, this issue), will support JAXA's Hayabusa 2 mission to investigate the C-type asteroid (162173) Ryugu that is expected to be a rubble-pile, with a diameter of $900 \mathrm{~m}$, thus slightly larger than Itokawa $(500 \times 300 \times 200 \mathrm{~m})$. The German Aerospace Center (DLR) developed MASCOT with contributions from CNES (France) (Jaumann et al. 2013; Ulamec et al. 2014; Grundmann et al. 2015). MASCOT comprises a payload of four scientific instruments: camera, radiometer (Grott et al. 2016, this issue), magnetometer (Hercik et al. 2016, this issue) and a hyperspectral microscope (Bibring et al. 2016, this issue).

Main objectives of MASCOT are to in-situ map the asteroid's geomorphology, the intimate structure, texture and composition of the regolith (dust, soil and rocks), and the thermal, mechanical, and magnetic properties of the surface in order to provide ground truth for the orbiter remote measurements, support the selection of sampling sites, and provide context information for the returned samples. C- and D-type asteroids hold clues to the origin of the solar system, the formation of planets, the origins of water and life on Earth. The mission will also provide information relevant for the protection of Earth from impacts, and resources for future human exploration. C- and D-types are dark and difficult to study from Earth (e.g. Vilas 2008), and have only been glimpsed by spacecraft. While results from recent missions (e.g., Hayabusa, and NEAR, e.g. Fujiwara et al. 2006; Abe et al. 2006; Saito et al. 2006; Bell and Mitton 2002) have dramatically increased our understanding of asteroids, important questions remain. For example, characterizing the properties of the regolith in-situ would deliver important ground truth for further understanding telescopic and orbital observations and samples of such asteroids. Hayabusa 2 is using an ion engine to travel to and rendezvous with the asteroid. The technology for Hayabusa 2 is inherited from Hayabusa with various improvements (Tachibana et al. 2014). During the 1.5-year 
Fig. 1 Artist's conception of Hayabusa 2 during sampling, also showing MASCOT landed on the surface.

CREDIT:JAXA/Akihiro Ikeshita

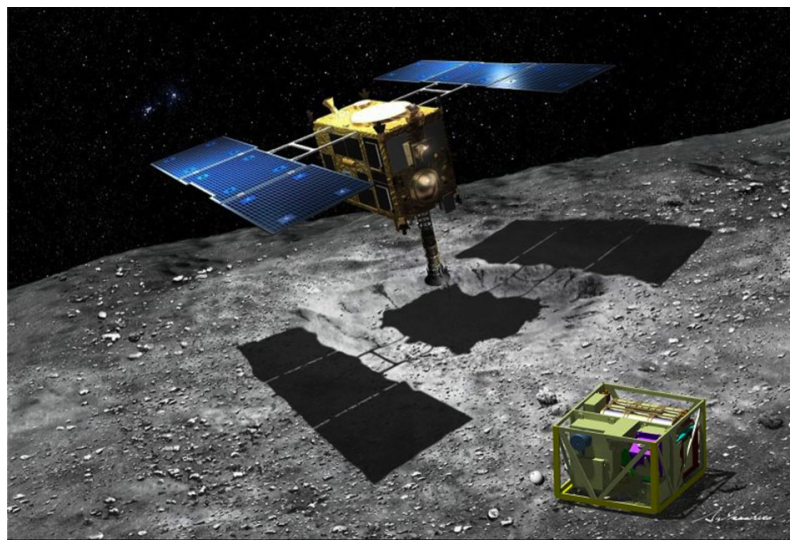

rendezvous period, Hayabusa 2 will investigate (1) the spin axis direction, (2) the asteroid shape, and (3) its surface characteristics (roughness, compositional features, and thermal properties) in the early survey phase. Once "the map" is created, touch down operations will be planned. The release of MASCOT will be coordinated with the touch down operations for sampling (Fig. 1).

\section{MASCOT Scientific Context}

The Hayabusa 2 mission will perform the first in-depth and close-up examination of near Earth asteroid (162173) Ryugu. This object has been classified as a Cg-type (Binzel et al. 2001) in the Small Main-Belt Asteroid Spectroscopic Survey classification system (Bus and Binzel, 2002a, 2002b), which is within the C-class in the Tholen system (Tholen and Barucci 1989). C-class objects are present in the inner belt (within $2.5 \mathrm{AU}$ ) but are most abundant in the middle to the outer main belt. They are believed to be primitive, volatile-rich remnants from the early solar system (Campins et al. 2013). The asteroid's shape has a small elongation, with an $a / b$ ratio of 1.12 (Kim et al. 2013). Its visible geometric albedo is $P_{v}=0.07 \pm 0.01$, its diameter is $0.87 \pm 0.03 \mathrm{~km}$, and its thermal inertia ranges between 200 and $600 \mathrm{Jm}^{-2} \mathrm{~s}^{-0,5} \mathrm{~K}^{-1}$ (Hasegawa et al. 2008; Campins et al. 2009; Müller et al. 2011). The thermal inertia is a factor 2 lower than that of 25143 Itokawa (Fujiwara et al. 2006) which indicates that the surface lies somewhere between a thick dust regolith and a rock/boulder-cm-sized, gravel-dominated surface (Hasegawa et al. 2008; Müller et al. 2011). The latter authors also report a retrograde sense of rotation with a spinaxis orientation of $\lambda_{\text {ecl }}=73^{\circ}, \beta_{\text {ecl }}=-62^{\circ}$ and $P_{\text {sid }}=7.63 \pm 0.01 \mathrm{~h}$. Spectral observations in July 2007 yield an absorption feature centered near $0.7 \mu \mathrm{m}$, and associated with the presence of iron-bearing phyllosilicates (Vilas 2008). In September 2007 a shallow absorption feature centered near $0.6 \mu \mathrm{m}$ was also observed by Vilas (2008). In contrast, the reflectance spectrum of Ryugu obtained during its discovery in 1999 showed no absorption feature (Binzel et al. 2002). Lazzaro et al. (2013) did also not detect spectral features out of $70 \%$ of the surface. These spectral observations suggest the asteroid's surface to be composed of different geological units. MASCOT will enable measurements during descent, at the landing and hopping positions, and during hopping. The first-order scientific objectives for MASCOT are to investigate at least at one location: 


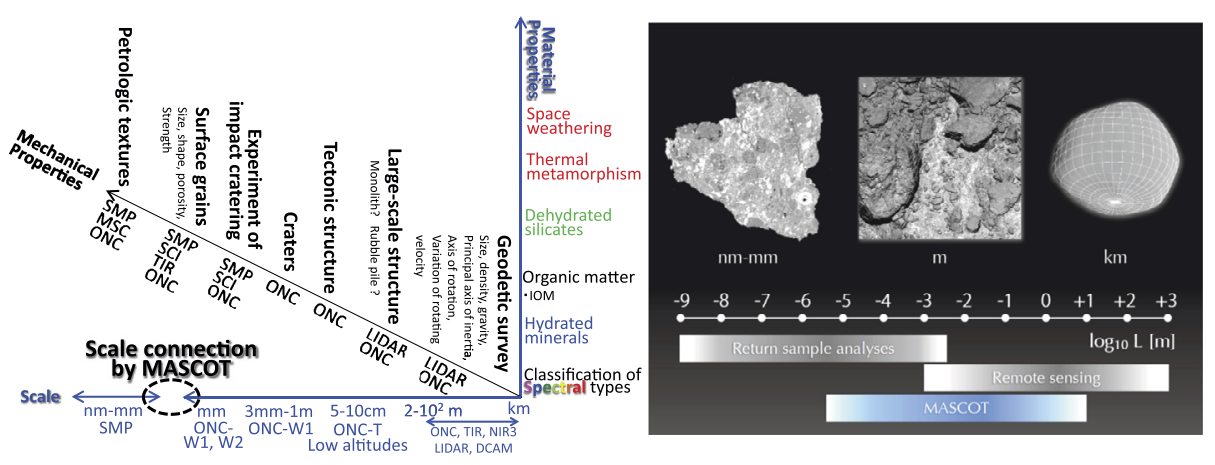

Fig. 2 Hayabusa 2/MASCOT combined measurement scales and addressed scientific investigations (modified from Sugita et al. 2013)

- the geological context of the surface by descent imaging and far field imaging in-situ;

- the global magnetization by magnetic field measurements during descent and any local magnetization at the landing positions;

- the mineralogical composition and physical properties of the surface and near-surface material including minerals, organics and near-surface ices;

- the surface thermal environment by measuring the asteroids surface temperature over the entire expected temperature range for a full day-night cycle;

- the regolith thermophysical properties by determining the surface emissivity and surface thermal inertia;

- the local morphology and in-situ structure and texture of the regolith including the rock size distribution and small-scale particle size distribution;

- the context of the observations performed by the instruments onboard the main spacecraft and the in-situ measurements performed by MASCOT ('coordinated MASCOT and Hayabusa 2 observations'), and the correlation of the local context of the in-situ analysis and the remotely sensed global context;

- the surface and possibly sub-surface physical properties on local and regional scales;

- the context of the sample collected and returned by the main spacecraft by qualifying its generic value and processed/pristine state and thus support the laboratory analysis by indicating potential alteration during cruise, atmospheric entry and impact phases.

Combined Hayabusa 2 and MASCOT visual and spectral observations will cover a wide range of observational scales. The MasCam observations, combined with the MASCOT hyperspectral microscope and MASCOT radiometer spectral observations, will serve as a strong tie point between Hayabus 2's remote sensing science $\left(10^{3}-10^{-3} \mathrm{~m}\right)$ and sample science $\left(10^{-3}-10^{-6} \mathrm{~m}\right)$ (Fig. 2) (Sugita et al. 2013; Jaumann et al. 2013; Ulamec et al. 2014; Grundmann et al. 2015; Schmitz et al. 2015).

\section{Camera Science Objectives}

The MasCam will provide the ground truth for the orbiter remote sensing observations. In addition, it will provide context for measurements by the other lander instruments (radiome- 
ter, spectrometer, and magnetometer), and the orbiter sampling experiment. By imaging during descent and on the surface, it will characterize the geological context, mineralogy and physical properties of the surface (e.g. rock and regolith particle size distributions). During day, clear filter images will be acquired. During night, illumination of the dark surface will be realized by four arrays of monochromatic light emitting diodes working in four spectral bands to allow identifying spectral variations on the surface. Continued imaging during the surface phase and image series at different sun angles over the course of an asteroid day will also contribute to the physical characterization of the asteroid surface properties by time-dependent photometric measurements of the regolith.

MasCam will contribute to the determination of the structural, textural and compositional characteristics of the surface layer by means of panchromatic and multi-color imaging of the asteroid surface on scale lengths ranging from tens of meters to a fraction of a millimeter. MasCam will operate as descent and in-situ imager (both during day and night phases). During descent, imaging will start shortly after the separation from the Hayabusa 2 mother spacecraft. Descent images will be acquired until touchdown of MASCOT. The images will close the resolution gap between orbital and surface imaging and allow to identify the landing site within the orbiter camera dataset. After touchdown, the camera will acquire wideangle images of the asteroid's surface. From these images, surface features will be mapped on scales ranging from meters down to a sub-millimeter to characterize the surface in terms of regolith physical properties, texture, morphology, particle size distribution, and microcraters. Multispectral imaging will classify and map the compositional heterogeneity of the asteroid's surface in order to spatially support the spectrally resolved information. The study of the spectral slope and albedo will allow to classify the solid surface phases, and to distinguish between carbonaceous, silicates and organic materials. Furthermore, the analysis of color ratios over a given field will provide information on the degree of soil heterogeneity at very small scales. Image series at different sun angles over the course of a day will also contribute to the physical characterization of the asteroid surface properties by photometric analysis. The images will also ideally guide the selection of sampling spot(s) of the Hayabusa 2 spacecraft, along with other results from the MASCOT in-situ measurements. Simultaneous observations with the spectrometer and the radiometer of the same surface location will provide comprehensive context information.

Additional imaging during the ballistic hopping and lander relocation mode will allow acquiring meso-scale (resolution: $\mathrm{cm}$ ) information of the local surface and/or possible indications of horizon glow that can not be obtained by the orbiter's imaging system.

The following measurement objectives apply for MasCam:

- investigation of surface features on scales ranging from meters down to a millimeter (regolith physical properties, texture, morphology, microcraters) through descent and closeup imaging, and photometry;

- determination of the rock fragment and particle size distribution of the regolith down to scales in the order of a millimeter;

- Identification of compositional and textural small-scale heterogeneities through color imaging in four spectral channels during dark phases;

- support of the selection of the sampling area by local characterization of candidate sampling sites;

- provide in-situ geological context of the asteroid's surface as:

- ground context for orbital measurements of Hayabusa 2

- context for all other MASCOT in-situ measurements. 


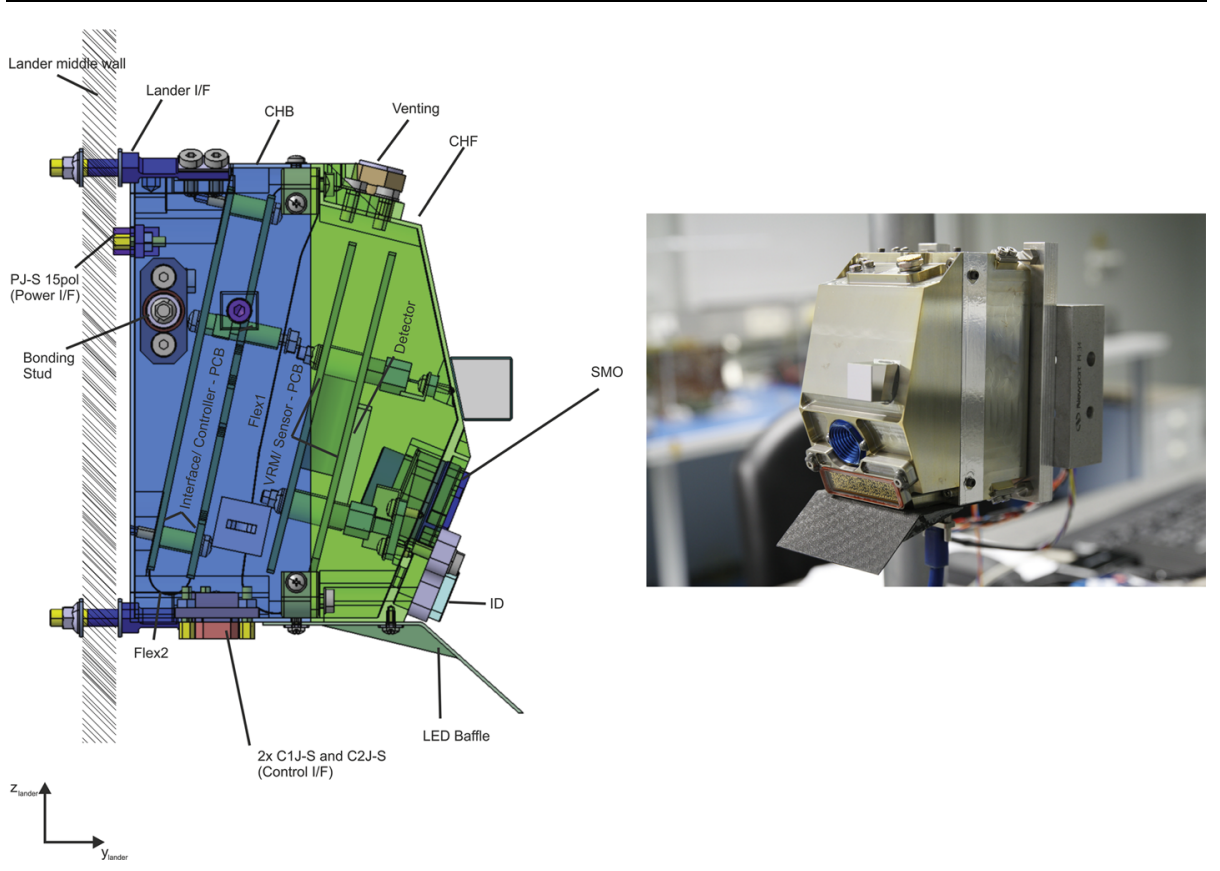

Fig. 3 Left: MASCOT drawing $(\mathrm{CHF}=$ Camera Housing Front, $\mathrm{CHB}=$ Camera Housing Back $\mathrm{SMO}=$ Surface mode optics, ID = Illumination Device, C1J-S,C2J-S,PJ-S = plug definitions). Right: MasCam flight model

\section{Instrument Description}

MasCam (Fig. 3) is a compact wide-angle Complementary MetalOxideSemiconductor (CMOS) camera, which has been designed to image a large part of the asteroid's surface in the field of view (FOV) of MASCOT. The lower front side of the camera, housing contains the optics, which has an optical axis that is slightly tilted towards the surface, such that the center of the camera's $54.8^{\circ}$ square FOV is located at an angle of $22^{\circ}$ with respect to the surface plane. This is to ensure that both the surface close to the lander and the horizon are in the FOV, when the lander rests horizontally on the surface after touch-down. The MARA radiometer FOV overlaps with the MasCam FOV, in order to correlate the radiometer's surface temperature information with visual information of the camera.

The optical layout of the camera is designed according to the Scheimpflug principle (Scheimpflug angle: $7.395^{\circ}$ ) with an aperture ratio of $f / 16$, so that the entire scene along the camera's depth of field, from $150 \mathrm{~mm}$ to the local horizon, is in focus. The camera utilizes a $1024 \times 1024$ pixel radiation-tolerant CMOS image sensor, which is sensitive in the 400-1000 nm wavelength range, peaking at 600-700 nm. Together with the $14.8 \mathrm{~mm}$ focal length, this yields a nominal ground resolution of $150 \mathrm{micron} / \mathrm{px}$ at $150 \mathrm{~mm}$ distance. An LED array, equipped with $4 \times 36$ LEDs of four different colors, centered at $470 \mathrm{~nm}$ (Blue), $530 \mathrm{~nm}$ (Green), $640 \mathrm{~nm}$ (Red), and $805 \mathrm{~nm}$ (NIR) illuminates the near field of the surface at night for color imaging (Fig. 4).

To satisfy the limited payload volume and mass allocation for the MASCOT payload, the camera optical head including illumination unit and electronics is designed to fit in a volume of $112.5 \times 136,0 \times 80.5 \mathrm{~mm}^{3}$ with a mass of only $407 \mathrm{~g}$. The typical power consumption is $1.5 \mathrm{~W}$ for panchromatic imaging, and max. $6.5 \mathrm{~W}$ for nighttime color imaging with the 

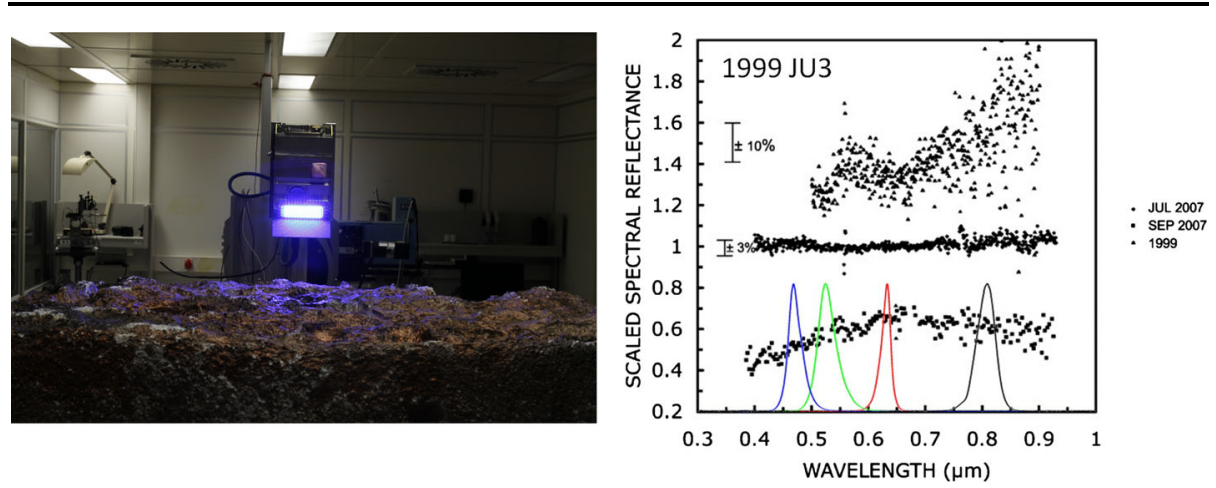

Fig. 4 MasCam with activated blue LED bank (left), and normalized spectral reflectance of the LEDs, drawn over the observations of asteroid 1999JU3 now named (162173) Ryugu (Vilas 2008) @ AAS (right).

LEDs. The camera flight model has undergone standard radiometric and geometric calibrations both at the component and lander-system levels. In-flight calibration of dark current is enabled by a calibration target mounted in the MasCam (and MARA) FOV during cruise.

MasCam uses a CNES-provided wavelet compression algorithm to maximize the data return within the stringent mission downlink limits. All calibration and flight data products will be archived in the Planetary Data System in the proper image format.

\subsection{Focal Plane and Sensor Electronics}

The functional blocks of the MasCam front-end electronics are shown in Fig. 5. MasCam consists of the following functional parts:

- Sensor Printed Circuit Board (PCB): OnSemi Star1000 CMOS Active Pixel Sensor (APS), analog chain +14 bit Analog-to-Digital Converter (ADC)

- Controller electronics PCB: FPGA Actel RTAX2000S + 16 Mb 3D plus SRAM

- Analog power conditioning PCB

- Interface electronics PCB: SpaceWire I/F (main/redundant), PCDU I/F, LED I/F, main power conditioning + filter.

The camera front end electronics is based on heritage from the ROLIS camera (Rosetta Lander Imaging System, Mottola et al. 2007), consisting of four printed circuit boards connected via rigid flex PCBs. The I/F PCB provides connection to all external interfaces, like the OnBoard Computer (OBC) and the Power Control and Distribution Unit (PCDU). Furthermore, it provides circuitry and connection to the illumination device. MasCam is controlled by a Field-Programmable Gate Array (FPGA) manufactured by Actel, which is located on the controller PCB together with a $16 \mathrm{Mb}$ SRAM from 3Dplus. It generates all pulses to control the Star1000 CMOS APS and its readout, and supervises data control and redundant data transfer plus failure detection to the OBC by implementing a SpaceWire protocol. Furthermore, it provides high-level algorithms for auto-exposure and engineering acquisition modes (e.g. a test pattern, which generates synthetic images). Additionally, the FPGA controls the illumination device by powering the different LED colors individually. Beside the sensor PCB, which accommodates the Star1000 APS (Fig. 6), the analog chain and a 14 bit ADC, an additional board hosts all analog power regulators for the analog circuitry that need to be separated from the digital part. 

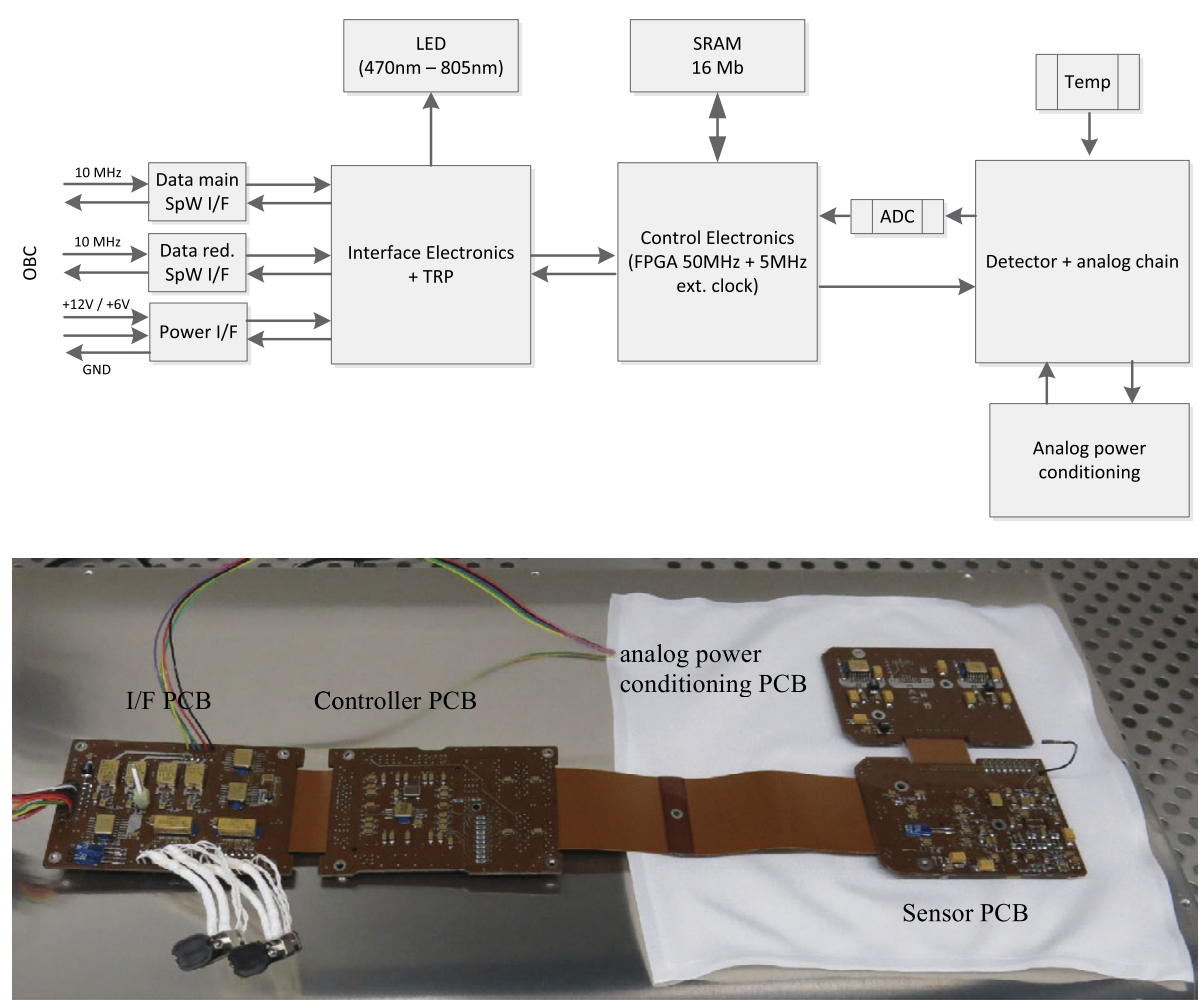

Fig. 5 MasCam front-end electronics block diagram and front end electronics PCB (backside)

Fig. 6 Sensor PCB with OnSemi Star1000 CMOS APS detector and 14 bit ADC

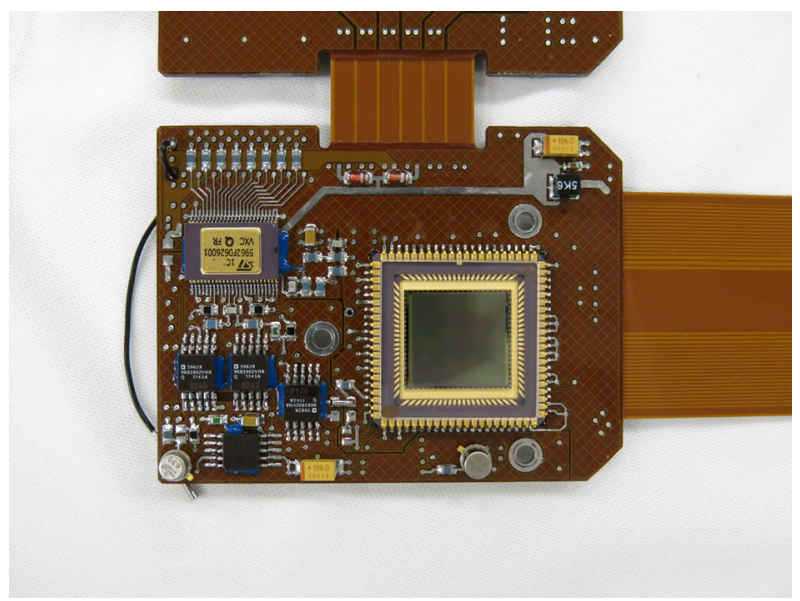

The CMOS Sensor and FPGA circuitry is based on an existing design from the PanCam High Resolution Camera (HRC) experiment for the ESA ExoMars Mission, whereas the complete analog chain with its 14 bit ADC was specifically developed for this project. The CMOS detector is a front-illuminated OnSemi Star1000 radiation-hard APS with a 3-T pixel 
Table 1 Optics design requirements

\begin{tabular}{ll}
\hline Item & Specification \\
\hline Focal length & $14.8 \mathrm{~mm}$ \\
Aperture & $\mathrm{f} / 16$ \\
Field of View (diameter) & $72.5^{\circ}$ \\
Spectral range & $400 \mathrm{~nm}-870 \mathrm{~nm}$ \\
Angular magnification & 1.0076 \\
Optical performance & diffraction limited, PSF $<30 \mu \mathrm{m}$ \\
Distortion & $<1 \%$ \\
Thermal range & working: $-55^{\circ} \mathrm{C} . .+80{ }^{\circ} \mathrm{C}$, survival: $-80^{\circ} \mathrm{C} . .+100{ }^{\circ} \mathrm{C}$ \\
Thermal stability & thermal focus shift within $\pm 30 \mu \mathrm{m}$ distortion stability within $\pm 0.1 \%$ \\
Mission radiation environment & 56 krad \\
Transmittance & Above $75 \%$ \\
Vignetting & no lens vignetting allowed \\
Ghosts & relative ghosts irradiance $<10^{-3}$ \\
\hline
\end{tabular}

architecture and an on-chip Fixed-Pattern Noise (FPN) correction. The device consists of a $1024 \times 102415 \mu \mathrm{m}$-pixel array with n-well/ p-substrate junctions for the photodiodes and an internal 10 bit ADC. In order to increase the available dynamic range it was decided to use an external 14 bit ADC to digitize the sensor signal. The electronic gain of the analog chain was set to $7.5 \mathrm{e}-/ \mathrm{DN}$ enabling digital saturation level to be reached slightly below the full well charge of the image sensor.

The sensor is operated in rolling-shutter mode where the image is not exposed simultaneously over the whole field but each line in the array is exposed sequentially. This process runs continuously when the camera is switched on, using an exposure time that is either pre-set or can be commanded. The sensor control circuitry is clocked at $5 \mathrm{MHz}$ leading to a minimum exposure time of $213.8 \mu$ s. For read out the image is directly transferred and stored to a $16 \mathrm{Mb}$ RAM from where the data are subsequentially transmitted with $10 \mathrm{Msps}$ via a redundant SpaceWire Link to the on-board computer (OBC).

\subsection{Opto-mechanical and Thermal Design}

MasCam follows three main scientific goals that are realized by its optical design:

- panchromatic imaging of the landing site and its vicinity during the descent.

- detailed multispectral and photometric investigations in high resolution ( 3 colors + NIR) of the asteroid surface in front of the Lander (nominal surface distance of CAM: $271 \mathrm{~mm}$ after touchdown of MASCOT).

- wide angle images during hopping (allowing CAM to look at different areas on the asteroid surface).

MasCam's wide-angle optics provides field-of-view (FOV) of $54.5^{\circ}\left(72.5^{\circ}\right.$ diagonal), which translates to an image scale of about $1.014 \mathrm{mrad}$ per pixel $(209.05 \% / \mathrm{px})$. The FOV requirement and therefore the whole optics design was driven by the geometry of the lander structure, which defined the maximum possible unobstructed FOV for the camera.

The optics design and manufacturing was performed by FISBA - Optik AG and based on the requirements listed in Table 1. 

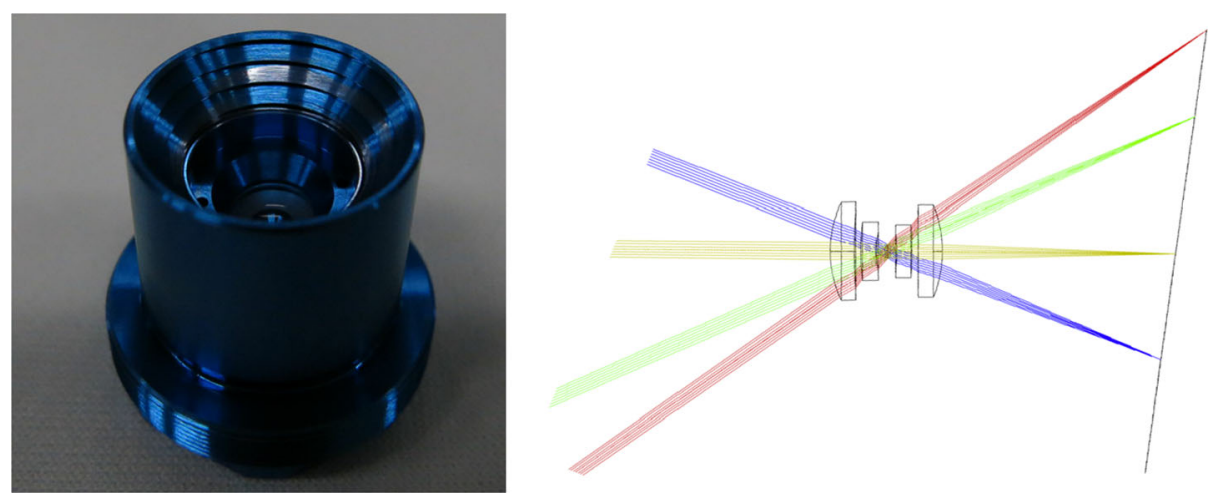

Fig. 7 MasCam optics (left) and optical design (right)

The refractive optics uses a four-lens Double Gauss design that provides, due to its symmetry, the inherent advantage of having very small optical aberrations (Fig. 7). The distortion of the lens is limited to less than $1 \%$. To maintain the transmittance and therefore optical performance of the optics during the mission timeline with a calculated total ionizing dose of $56 \mathrm{krad}$, all glasses are made from the radiation-tolerant materials LAK9G15 and F2G12. The relative illumination of the optics over the FOV is close to the corresponding characteristics of a perfect lens, where over the FOV the relative illumination decreases according the "cosine fourth" law of illumination falloff.

The entire envelope was restricted to a max. diameter of $22 \mathrm{~mm}$ and a length of $22 \mathrm{~mm}$. In order to cope with the thermal stability requirement within the given temperature range the lens barrel and most of the mechanical components are made from Titanium (CTE $8.610^{-6} \mathrm{~K}^{-1}$ ). Thus, a focal shift of $20 \mu \mathrm{m}$ over $\Delta \mathrm{T}$ of $135 \mathrm{~K}$ could be reached having an overall mass of $<16 \mathrm{~g}$. A baffle system in front and on the backside of the lens system allows unaffected measurements even with the sun at $45^{\circ}$ off the optical axis.

The optics has a focal length of $14.8 \mathrm{~mm}$ with an f-Number of 16 leading to a sufficient field depth. In order to compensate for the $22^{\circ}$ camera tilt and to maintain the optimal depth of field by putting the entire FOV into focus, the sensor optical axis has been tilted by $7.395^{\circ}$ towards the optical axis (Scheimpflug design principle). Furthermore, the center of the sensor is shifted by $1 \mathrm{~mm}$ with respect to the optical axis.

The sensor PCB provides three holes (Fig. 8) arranged symmetrically around the sensor optical axis to allow an isostatic mounting to compensate the mismatch in the coefficient of thermal expansion (CTE) between the PCBs, the front side housing of aluminum and the lander's structure (Carbon-Fiber-Reinforced Polymer (CFRP) sandwich). The isostatic mounting is realized by three flexure-mounts made of Ti-Al6-4V. The sensor alignment was achieved by using shims made of Titanium. The L-shaped baffles along the flex-mounts were used for stray light reduction. For further straylight reduction, all inner parts of the camera housing were coated with Keplacoat-black ${ }^{\circledR}$.

The flexure mounts are located in the corners of the MasCam housing such that the centroid of the formed triangle is located in the geometrical center of the camera housing. Thus, thermally induced stress and strain to the housing was avoided and the camera alignment will be stable over the mission timeline.

The current mission scenario expect landing on Ryugu when the asteroid is at a heliocentic range of about $1 \mathrm{AU}$, corresponding to a solar irradiance of $\sim 1360 \mathrm{~W} / \mathrm{m}^{2}$. Based on the current thermal model this will lead to environment temperatures between $+60{ }^{\circ} \mathrm{C}$ (landing 

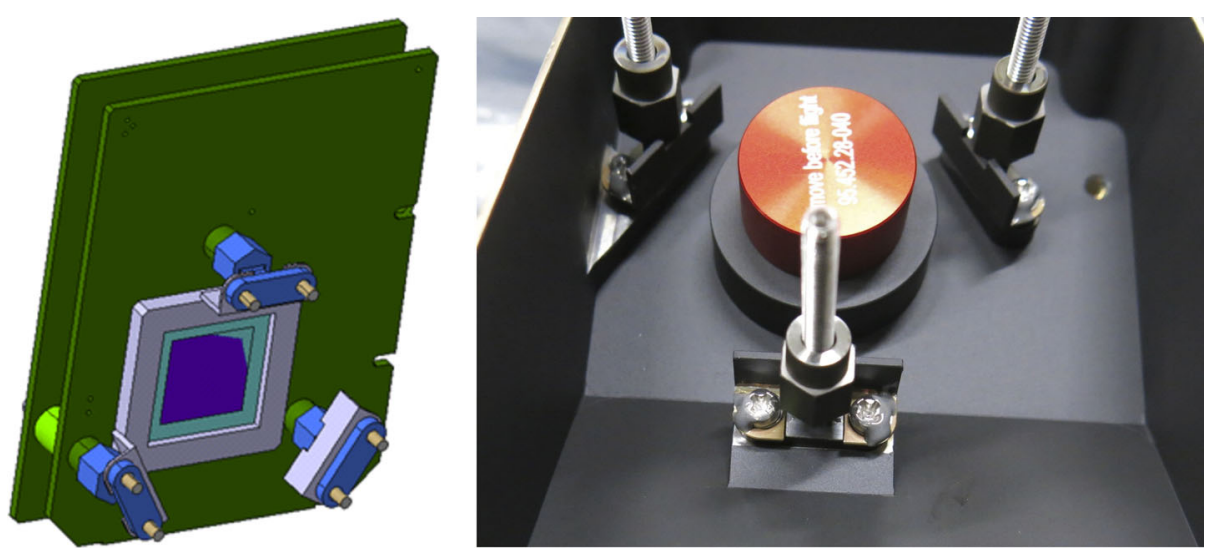

Fig. 8 Sensor PCB flexure mount (front and back)

hot case) and $-55{ }^{\circ} \mathrm{C}$ (landing at night, cold case). The main design drivers were a passive thermal control of the instrument, and the thermal isolation of MasCam from the lander. Thus, the relevant requirements were to keep the instrument temperature stable within the operational design limits and to minimize thermal heat flow to the lander unit. In order to fulfill these requirements, the approach to the thermal design was twofold: first, the camera housing was thermally isolated from the lander structure by the flexure mounts, which, as described above, are made from Titanium with a low thermal conductivity $(7.4 \mathrm{~W} / \mathrm{mK})$. Together with the small cross sectional area, this leads to a relatively high thermal resistance of $\sim 310 \mathrm{~K} / \mathrm{W}$ per mount, isolating the camera housing from the lander. Second, the emissivity of the camera housing's surface was reduced by applying an alodine coating.

During cruise, the thermal model predicts a camera temperature between $-55^{\circ} \mathrm{C}$ and $+20{ }^{\circ} \mathrm{C}$, which is well compliant with the required non-operational temperature range. The temperature on the surface of the asteroid is strongly depending on the location of the landing site but also on the orientation of the lander towards the sun. Thus, the coldest temperature during operation corresponds to $-58{ }^{\circ} \mathrm{C}$ when the lander is facing south. The hottest case occurs when the lander is orientated towards west, with worst-case temperatures of $+95{ }^{\circ} \mathrm{C}$ near the end of the mission. It is currently planned to cope with these uncertainties mainly by operational means but also by iterating and updating the current thermal model of Ryugu.

The thermal mathematical model of MasCam inside the MASCOT lander was developed by Airbus DS using ESATAN and ESARAD. The model is composed of 254 nodes, 487 conductive- and 10900 irradiative links, including the asteroid environment and deep space. The model serves to calculate the steady-state thermal environment, but is mainly used for calculating transients including the operation on the asteroid surface.

To monitor and control the instrument thermal environment MasCam is equipped with two redundant thermistors that are used as thermal reference points (TRP). Therefore, the temperature readings at these TRPs are used to determine whether the camera is within its operational temperature range and can be switched on. These thermistors (PT-1000 elements) are directly routed to the MASCOT On-Board Computer (OBC), such that temperature readings can be obtained at all times during MASCOT lander operations.

In addition to the redundant TRPs the camera is equipped with an additional thermistor located directly below the imaging sensor. The sensor is multiplexed, digitized and read out 
by the camera electronics and readings are subsequently transmitted as part of the housekeeping data to the $\mathrm{OBC}$.

\subsection{Spectral Illumination Unit}

An LED array, equipped with $4 \times 36$ LEDs of different colors (centered at $470 \mathrm{~nm}$ (blue), $530 \mathrm{~nm}$ (green), $640 \mathrm{~nm}$ (red), and $805 \mathrm{~nm}$ (NIR)), is available to illuminate the surface at night for color imaging. During instrument calibration, the spectral bands of the four LED colors were determined by illuminating the inside of a small integrating sphere at a distance of $7.8 \mathrm{~cm}$, measured perpendicular to the LED array (Fig. 4).

\subsection{Calibration Target}

An in-flight calibration target for MasCam dark-current and MARA temperature calibration is mounted inside the MasCam and MARA FOV during cruise. The calibration target will stay on the orbiter after separation. The calibration target has two dedicated areas. The larger area, which is mounted in front of the MasCam's FOV, is designed to allow for in-flight calibration of the MasCam's dark current. For this purpose, the surface is coated with Fractal Black. The payload compartment, where MASCOT is accommodated inside Hayabusa 2, is situated behind Hayabusa 2's solar panels. Therefore, the compartment is dark during cruise. Thus, the calibration target can also be used in order to check the functionality of the LED illumination unit, by illuminating its dark surface. The area in front of MARA's FOV is macro-structured to achieve emissivity near infinity. For MARA temperature calibration, the target can be temperature-controlled for in-cruise calibration checks and possible recalibration.

\section{Mass, Power, Data, Environment}

MasCam was designed to cope with the requirements imposed by the limited resources of volume and mass for MASCOT inside Hayabusa 2. Without attachments and grounding provisions, the main body has a volume of $81 \times 109 \times 67 \mathrm{~mm}^{3}$. The camera flight model has a mass of $407 \mathrm{~g}$ (design target was $440 \mathrm{~g}$ without margin).

MasCam is powered through the MASCOT PCDU, which provides two regulated voltage lines to the camera. The main input line for the camera electronics is the $6 \mathrm{~V}$ line $(5.8 \mathrm{~V}-$ 6.2 V DC). Due to the sensitivity of the imaging sensor to voltage fluctuations requires the $6 \mathrm{~V}$ to be delivered highly stabilized with a maximum ripple of $25 \mathrm{Vpp}$. A second line, providing $+12 \mathrm{~V}(11.5 \mathrm{~V}-12.5 \mathrm{~V} \mathrm{DC})$ is used to switch the LEDs on and off. The power budget ranges between 1.4-1.8 $\mathrm{W}$ for imaging and 4,2-6.4 $\mathrm{W}$ for imaging + LED. The power consumption mainly depends on the operational temperature and the operational modes of the camera.

The Star1000 imaging sensor consists of a $1024 \times 1024$ matrix of pixels, which are transmitted as 2-byte words. Thus, the data volume for one image has an uncompressed size of 2.097152 Mbytes. This volume is transferred with $10 \mathrm{Msps}$ to the MASCOT OBC, where the image post-processing and compression is performed.

The camera housekeeping (HK) provides the telemetry on the thermal situation of the instrument, the subsystem health. Each HK packet comprises 20 different parameters, which are transmitted to the OBC by telecommand. 
The shielding characteristics of the lander have been modeled with FASTRAD. All electrical components including the imaging sensor were selected based on their compliance to the determined radiation levels, including the mandatory design margin factor of two. Further the front-end electronics were designed such that the scientific performance of the instrument is not compromised by single event effects, latch-ups, but also derating effects.

The mechanical design of the camera was developed to withstand the mission environment during its lifetime, including transportation, launch, cruise and landing. MasCam is further designed to reduce as far as possible detrimental influences toward the lander's or other instruments' performance. The highest loads were expected to occur during the launch with the in terms of high frequency, and random vibration loads. The loads experienced by MasCam are also influenced by resonances of the lander structure and the Hayabusa 2 structure. In order to account for this, the camera was qualified with vibration levels up to $17.49 \mathrm{~g}_{\mathrm{RMS}}$.

To allow for a pressure decay of $5 \mathrm{kPa} / \mathrm{s}$ during ascent, the camera housing is equipped with venting provisions allowing the depressurization during launch, but also ensure the required dust tightness to prevent a contamination during on-ground testing and landing.

\section{Integration}

The integration of MasCam was performed at Airbus DS GmbH in Munich. A cleanroom (ISO class 5) was used to assemble the different camera elements (Fig. 9). The optical alignment and test setup was located on an optical bench in an ISO class 8 clean room. After the first integration with nominal shims installed under the detector PCB, a set of measurements were performed to check the camera focus. Based on these measurements, shim corrections were performed by shimming rings between the optics and the housing, as well as between the detector PCB and the camera housing (on the kinematic mounts). After the final confirmation of the correct shim thickness under both the optics and the detector PCB, the full torque was applied to the different fasteners and the camera housing was sealed off.

Due to the structural concept of the lander's CFRP box, the payload compartment has no walls, but only CFRP struts (Fig. 10). In the direction of MasCam's FOV, a diagonal strut limits the possible FOV. The size of the camera's FOV (Fig. 11) is designed to just fit within the area left free by the diagonal strut and the sidewall. In order to guarantee proper alignment of the camera's FOV with regard to the lander structure, the interface between housing and mounts has been shimmed with thin washers. For alignment purposes, an aluminum alignment cube with four optical surfaces, coated with PVD-Al and $\mathrm{SiO}_{2}$, has been glued to the camera.

To shield MasCam from stray light from the magnetometer, a dedicated baffle was mounted on the MasCam underside where the magnetometer is covered in black MLI. All surrounding MASCOT struts have been kept free of capton tape, in order to further reduce any stray light source, and to avoid any possible reflection of the LED illumination on a MASCOT surface.

\section{Instrument Performance and Calibration}

A test and calibration program has been conducted to derive MasCam instrument performance and calibration parameters. These tests were conducted at the individual component level (imaging sensor with electronics, optics, LED illumination unit), at the assembled 


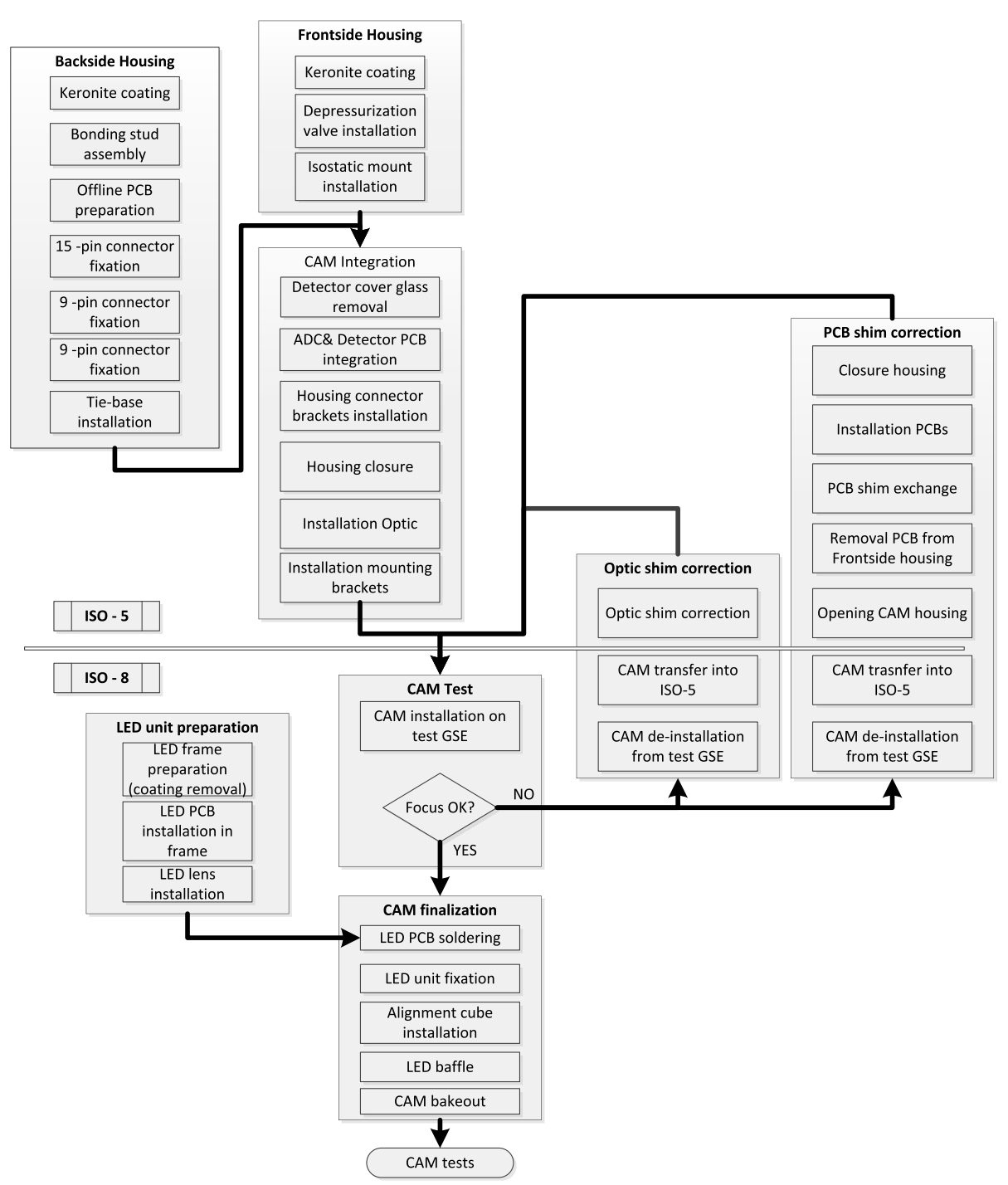

Fig. 9 MasCam AIT flow

(standalone) camera level for the flight and flight spare unit and finally at the system level during fully assembled lander testing. Tests were performed at both (Earth) ambient temperature and pressure conditions and under thermal vacuum conditions simulating the range of expected conditions during cruise, descent and surface phase.

\subsection{Component Level and Camera Standalone Tests and Geometric Calibration}

MasCam was geometrically calibrated at Airbus DS including focus stability tests. Geometric distortion, distortion stability, as well as in- and out-field stray light were also measured (Fig. 12) and confirmed to be within the design limits. To find the optimum focus position of the camera, a point source was imaged on the detector. The optimum point spread function 

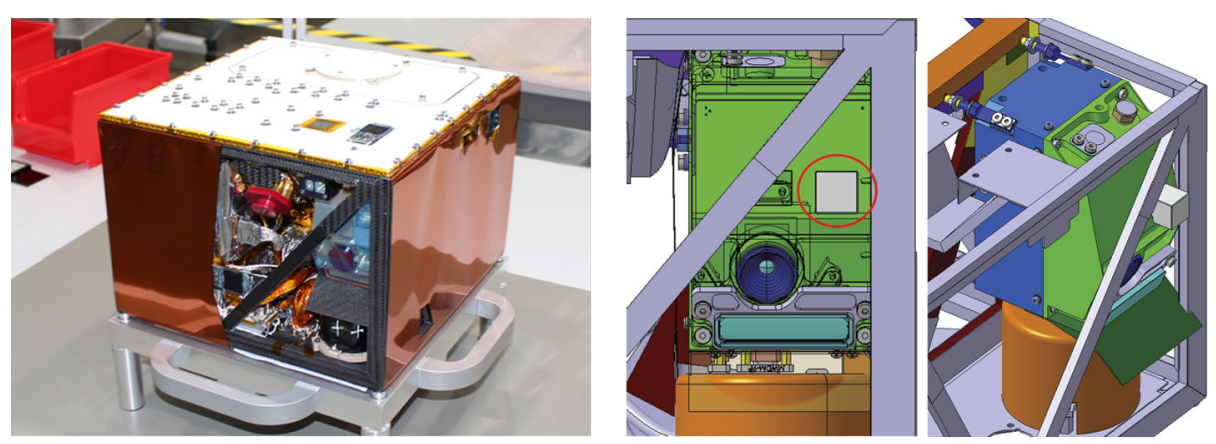

Fig. 10 MasCam integrated in Mascot (left) and MasCam's mounting position in MASCOT

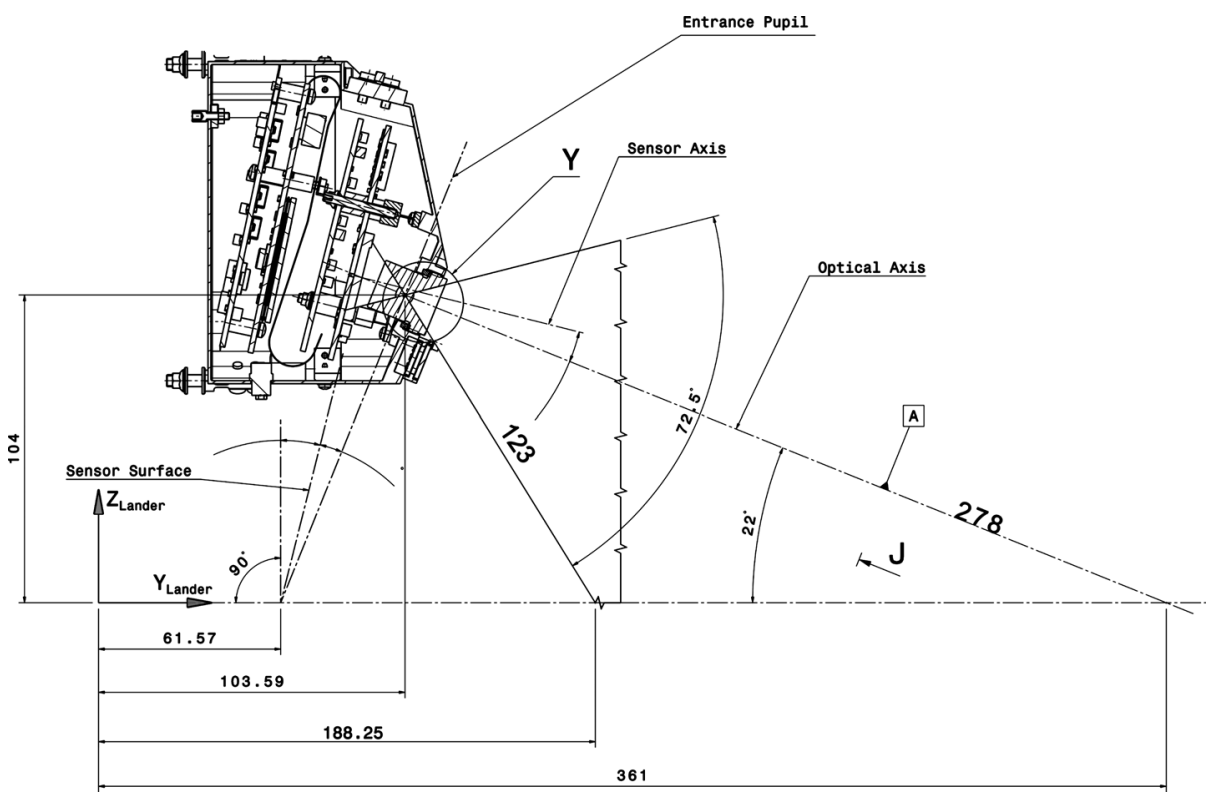

Fig. 11 MasCam's FOV

was found by changing the distance between light source and detector around its nominal value. The measured function fit to a Gaussian profile and the FWHM of that function was determined. The process was repeated for nine points distributed on the detector, thus taking the Scheimpflug configuration into account. This information was used to correct both the distance between detector and optics and the tilt of the detector. Independent shims allowed correction of these parameters independently. The focal length stability was verified over the complete operational thermal range during the thermal-vacuum cycling test campaign. Additional characterization tests were performed at the same premises to determine the resolution of the camera (measurement of Modulation Transfer Function (MTF)), the distortion of the optics, and the stray light behavior. For the distortion measurements, a checkerboard pattern was used, which was imaged by the camera. The generated distortion map will allow subsequent calibration of the camera raw images on ground. The MTF (Fig. 13) was determined using Siemens stars at different distances and at different positions on the detector. 

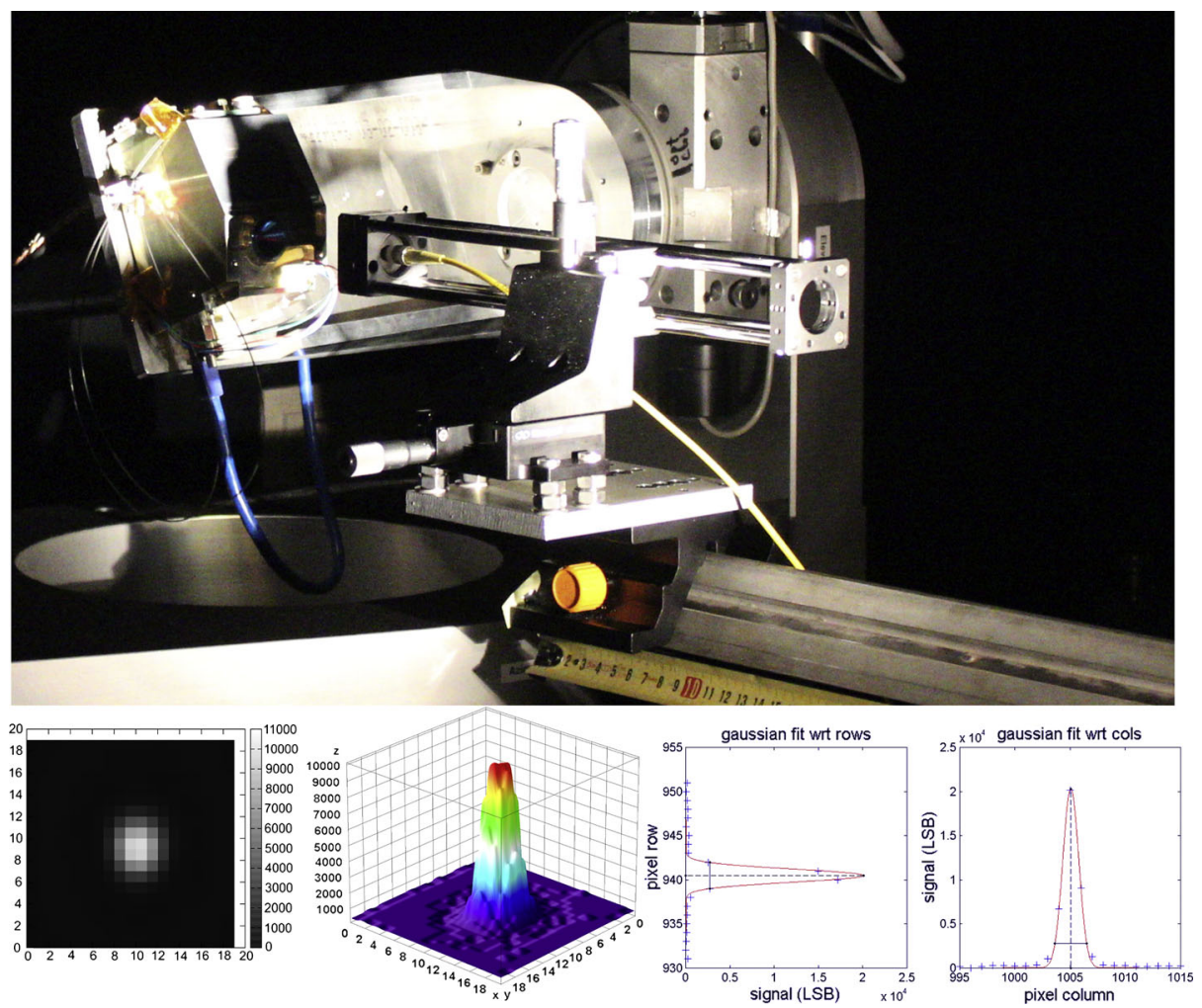

Fig. 12 Overview of the alignment setup (upper picture), Imaged point source to find the optimum focus position (lower left), 3D illustration (lower center) and fitted Gaussians in vertical/horizontal directions (lower right)
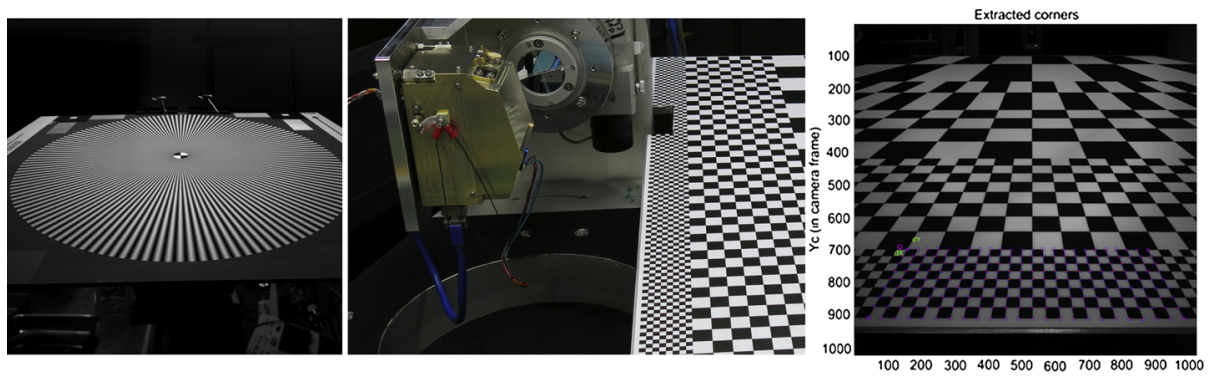

100200300400500600
Xc (in camera trame)

Fig. 13 Image Siemens star for MTF determination (left), overview of setup for camera distortion characterization measurement (center), Camera-imaged distortion test-pattern (right)

\subsection{Radiometric calibration}

Radiometric calibration was performed in the fall and winter of 2013. A similar campaign was performed for the flight spare (FS). The calibration pipeline for MasCam images contains the following steps: bias subtraction, correction for non-linearity, dark subtraction, flat field division. 
Fig. 14 The LED spectral flux at a distance of $7.8 \mathrm{~cm}$ from the LED array as determined with aid of a small integrating sphere

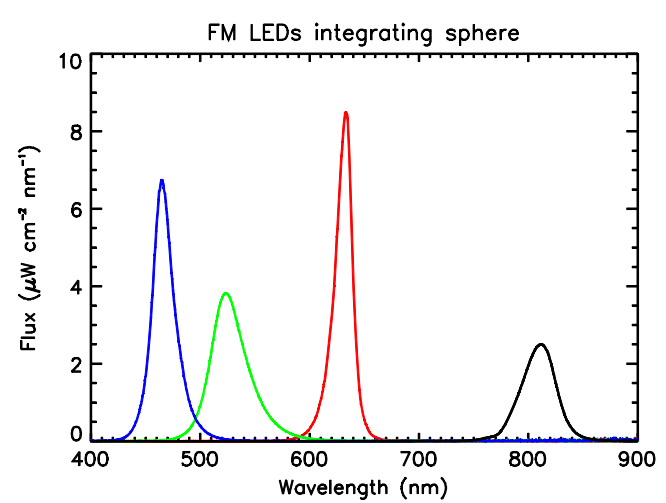

Table 2 Radiometric properties of the MASCOT camera LEDs. $R$ is the responsivity factor

\begin{tabular}{lllllc}
\hline$\#$ & LED & $\lambda_{\text {cen }}[\mathrm{nm}]$ & $\lambda_{\text {eff }}[\mathrm{nm}]$ & FWHM $[\mathrm{nm}]$ & $R\left[\mathrm{~m}^{2} \mathrm{sr} \mathrm{mJ}^{-1}\right]$ \\
\hline 1 & Blue & 465 & 471 & $455-477$ & $110.7 \pm 1.1$ \\
2 & Green & 523 & 532 & $508-544$ & $129.3 \pm 1.2$ \\
3 & Red & 633 & 630 & $623-640$ & $125.1 \pm 1.3$ \\
4 & $I R$ & 812 & 809 & $792-827$ & $97.1 \pm 1.1$ \\
\hline
\end{tabular}

These steps are described by:

$$
\mathbf{C}=[L(\mathbf{S}-\mathbf{B})-L(\mathbf{D}-\mathbf{B})] / \mathbf{F}
$$

where the $\mathbf{C}, \mathbf{S}, \mathbf{B}, \mathbf{D}, \mathbf{F}$ are the clean, raw, bias, dark, and flat field images, respectively (we reserve bold symbols for images). The dark image is acquired at the same exposure time as the raw image. $L(\mathbf{I})$ is the function that corrects image $\mathbf{I}$ for non-linearity. The $\mathbf{C}$ image is essentially a radiance image, but with arbitrary units. Images acquired with LED illumination (Fig. 14, Table 2) can be further calibrated to radiance and relative surface reflectance as explained below. At times we refer to the coordinates of a pixel within the CMOS frame. Pixel $(x, y)=(0,0)$ is the one in the lower left corner, where $x$ and $y$ are in the sample and line direction, respectively. LED color names are indicated in italics. The bias image (B in Eq. (1)) is defined as the image acquired at the shortest possible exposure time. An exposure time is defined as a number of steps, with a single step being $0.2138 \mathrm{msec}$, and the shortest exposure time is one step. The bias image represents the signal present on the CMOS at the start of an exposure, and must be subtracted as a first step in the calibration pipeline. Figure 15 shows a typical bias image. The median of this image is $372 \mathrm{DN}$, and the signal increases towards the left and right edges of the image. The bias is not constant and not a regular function of temperature. In a thermal vacuum test over a wide range of temperatures, the median bias was found to be $403 \pm 19 \mathrm{DN}$ ( $n=$ 13). The CMOS detector is non-linear. We distinguish two exposure time $\left(t_{\exp }\right)$ regimes with different non-linear behaviors.In the short regime the exposure times range from 1 to 1023 steps $(0.2138-218.7174 \mathrm{msec})$. The long regime has exposure times starting from 1024 steps $(218.9312 \mathrm{msec})$. The critical exposure time separating the two regimes is therefore $218.8 \mathrm{msec}$. To determine the non-linearity of the CMOS we took images of the inside of a large integrating sphere (IS). To assess the degree of linearity we measured the average signal in a small $(21 \times 21$ pixels $)$ square centered at $(x, y)=(512,600)$, or just above 
Fig. 15 Typical bias image. The such that black and white represent 250 and $600 \mathrm{DN}$, respectively. Note that the signal in some pixels exceed these display limits brightness of this image is scaled
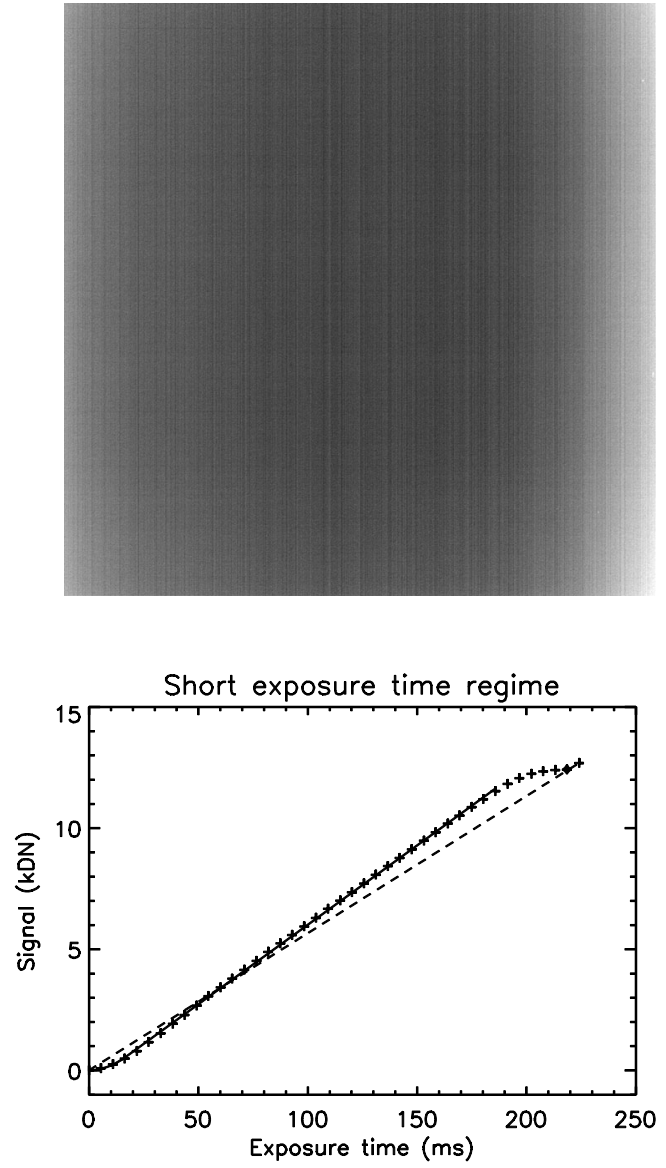

Fig. 16 Non-linearity of the CMOS detector in two exposure time ( $\left.t_{\exp }\right)$ regimes. Top Short regime $\left(t_{\exp }<218.8 \mathrm{~ms}\right)$. Bottom Long regime $\left(t_{\exp }>218.8 \mathrm{~ms}\right)$. The plus symbols are the observations, with the filled diamond representing the image with a commanded exposure time of 1023 steps. The drawn line is the model and the dashed line is the desired, linear behavior

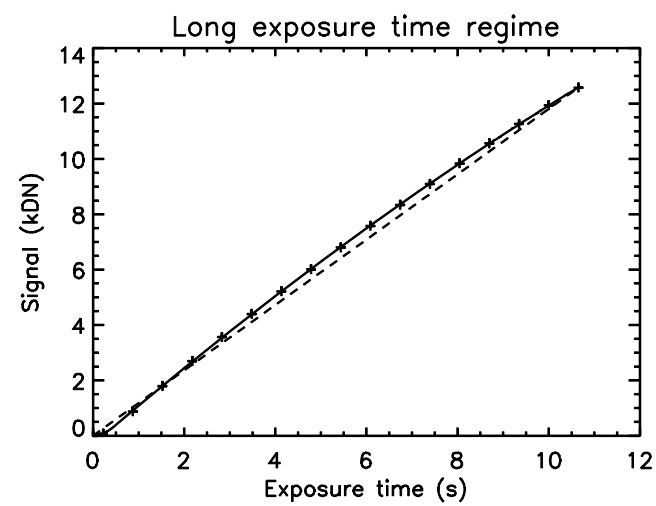

the image center. Figure 16 shows the observed signal for the short exposure time regime $\left(t_{\exp }<218.8 \mathrm{msec}\right)$. In the short regime the detector is approximately linear over a limited range, but with a slope that is different from that desired. For this particular brightness level of the IS the linear range extends over the 20-180 msec exposure time range. The figure includes one exposure with a time that exceeds 1023 steps. At lower exposure times the 
signal is slow to increase, and at higher exposure times the signal flattens out, giving the linearity curve a sigmoid shape. The flattening for exposure times toward 1023 steps is not related to saturation, as it is also observed for other parts of the CMOS where the signal levels were much lower. Therefore we conclude that the commanded exposure times in this range do not correspond to reality. We define the true signal by the dashed line in the figure, which connects zero with the signal at the exposure time of 1023 steps. The model for the observed signal is indicated by the drawn line. The model for the linear range for intermediate exposure times is a line, and a parabola for exposure times at the low end. The non-linearity correction converts observed signal $\left(S_{\text {obs }}\right)$ into corrected signal $\left(S_{\text {cor }}\right)$, and must be applied after bias subtraction. The correction for the short exposure time regime is

$$
\begin{aligned}
& S_{\text {cor }}=\left(1595 \times S_{\mathrm{obs}}\right)^{1 / 2} \mathrm{DN} \text { for } S_{\mathrm{obs}}<532 \mathrm{DN}, \\
& S_{\mathrm{cor}}=0.8654 \times S_{\mathrm{obs}}+461 \mathrm{DN} \text { for } 532 \mathrm{DN}<S_{\mathrm{obs}}<11.5 \mathrm{kDN}
\end{aligned}
$$

with $S_{\text {cor }}$ and $S_{\text {obs }}$ in DN. Currently we do not model the non-linearity of the exposure time towards 1023 steps, which means that signal levels over $11.5 \mathrm{kDN}$ in the short regime cannot be trusted. Figure 16 bottom shows the results for the long exposure time regime $\left(t_{\exp }\right.$ $>218.8 \mathrm{msec}$ ). The IS brightness level was lower than during the short regime experiment. In the long regime the detector is not linear over any range. Again we see that at lower exposure times the signal is slow to increase, but we do not observe flattening of the signal level at the highest exposure times. We define the true signal by the dashed line in the figure, which connects zero with the signal at the highest exposure time. The model for the observed signal is indicated by the drawn line. We model the signal with two connected parabolas. The non-linearity correction converts observed signal $\left(S_{\text {obs }}\right)$ into corrected signal $\left(S_{\text {cor }}\right)$, and must be applied after bias subtraction. The correction for the long exposure time regime is

$$
\begin{aligned}
& S_{\mathrm{cor}}=\left(S_{\mathrm{obs}}\right)^{1 / 2} \mathrm{kDN} \text { for } S_{\mathrm{obs}}<306 \mathrm{DN}, \\
& S_{\mathrm{cor}}=0.01311 \times\left(S_{\mathrm{obs}}\right)^{2}+0.8084 \times S_{\mathrm{obs}}+0.3055 \mathrm{kDN} \text { for } S_{\mathrm{obs}}>306 \mathrm{DN}
\end{aligned}
$$

with $S_{\text {cor }}$ and $S_{\text {obs }}$ in $\mathrm{kDN}$. At the operational temperatures on the asteroid we expect significant dark current accumulation. This needs to be subtracted from the raw image. Ideally this is done by acquiring a dark current image with the same exposure time as the original image (D in Eq. (1)). Note that this would only be possible during the asteroid night, as the MASCOT camera has no shutter. We characterized the CMOS dark current before launch as follows. The bulk dark current is defined as the median dark current of the full image frame. Before calculating the dark current the images were bias-subtracted and corrected for nonlinearity. Figure 4 shows the bulk dark current (defined as the median of the image frame excluding bad columns and rows) as determined from a series of images acquired in lab thermal vacuum in November 2013. The dark model we use to fit the data is the Arrhenius law:

$$
D(T)=a \exp \left[-b /\left(\mathrm{k}_{\mathrm{B}} \times T\right)\right]
$$

with $D$ the dark current in DN s${ }^{-1}$ for CMOS temperature $T$ in $\mathrm{K}, \mathrm{k}_{\mathrm{B}}=1.38065 \cdot 10^{-23}$ $\left[\mathrm{m}^{2} \mathrm{~kg} \mathrm{~s}^{-2} \mathrm{~K}^{-1}\right]$ the Boltzmann constant, and $\mathrm{a}$ and $\mathrm{b}$ two additional constants. The figure shows that the model fits the data very well with $a=1.28 \cdot 10^{16} \mathrm{DN} \mathrm{s}^{-1}$ and $b=1.33 \cdot 10^{-19}$ $\left[\mathrm{m}^{2} \mathrm{~kg} \mathrm{~s}^{-2}\right]$. Only at the very lowest temperature in the figure there is a mismatch between model and observations due an imperfect non-linearity correction, which appears to be not accurate for very low signal values. Therefore it was not possible to reliably determine the bulk dark current at lower temperatures than shown in the figure. This model represents the 
Fig. 17 The bulk dark current, defined as the median of the image frame, as a function of CMOS temperature. The images were corrected for bias and non-linearity. The dashed line is the best-fit dark current model

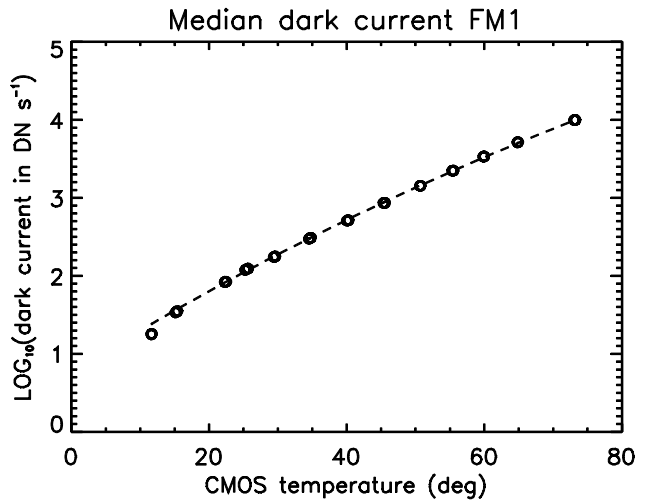

Fig. 18 The FM flat field

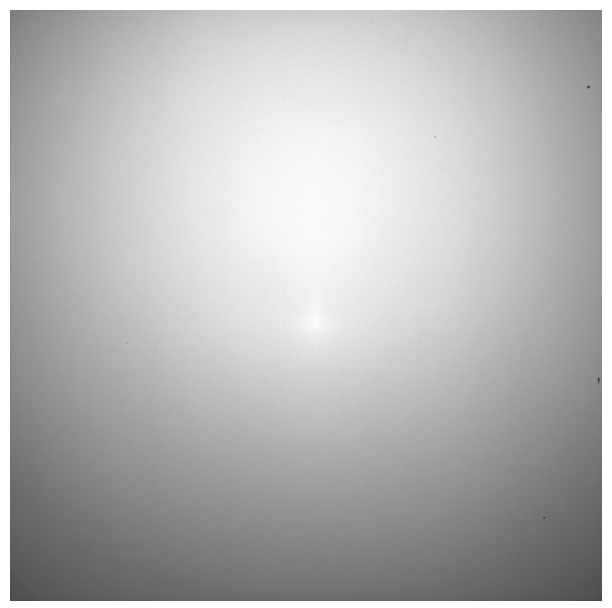

bulk dark current status at the time of November 2013. However, the dark current is expected to increase steadily during MASCOT's many years in space and will be significantly higher at the asteroid. Nevertheless, Fig. 17 can be used to make a coarse estimate of the expected dark current on the asteroid.

Hot pixels represent pixels with a dark current significantly higher than the bulk. To illustrate the prevalence of hot pixels we investigate one dark current frame reconstructed from a $4.90 \mathrm{sec}$ exposure at $40{ }^{\circ} \mathrm{C}$ in November 2013. The dark current mean \pm standard deviation $(\mu \pm \sigma)$ was $2638 \pm 864 \mathrm{DN}$. The fraction of pixels exceeding a dark current of $\mu+3 \sigma$ was $1.14 \%$. The fraction of pixels exceeding $\mu+6 \sigma$ was $0.07 \%$. A flat field (F in Eq. (1)) was constructed as the median of 20 images of the inside of an integrating sphere. The images were corrected for bias and non-linearity. The images were not corrected for dark current. The CMOS temperature was $33{ }^{\circ} \mathrm{C}$ and the expected dark current around $250 \mathrm{DN} \mathrm{s}^{-1}$, which corresponds to $1.5 \mathrm{DN}$ for the exposure time of the images $(6.0 \mathrm{msec})$. As the maximum image signal (in the center) was around $8 \mathrm{kDN}$, dark current can be considered negligible. Figure 18 shows the resulting flat field. It can be seen that the flat field is affected by strong vignetting. A small spot of enhanced brightness in the center may be due to infield stray light. Small dark spots are most likely particles on the CMOS surface. We assume the flat field to be valid over the full wavelength range of the LEDs, as it proved not possible to reliably construct flat fields for each of the LED colors. It is normalized to the average 
signal inside a small circle (6 pixels radius) centered at $(x, y)=(484,553)$, at the location of the Murchison pellet.

The absolute radiometric calibration of images that are acquired with LED illumination is given by

$$
\mathbf{I}_{i}=\mathbf{C}_{i} /\left(t_{\exp } \times R_{i} \times \mathbf{V}_{i}\right)
$$

for each of the four LED colors $i=(1,2,3,4)$ for (Blue, Green, Red, IR), with $\mathbf{I}$ the radiance image with units of [W $\mathrm{m}^{2} \mathrm{sr}^{-1}$ ], $\mathbf{C}$ the clean image from Eq. (1), $\mathbf{V}$ the color ratio image, $t_{\exp }$ the exposure time in sec, and $R$ the responsivity factor in $\left[\mathrm{m}^{2} \mathrm{sr} \mathrm{mJ}^{-1}\right]$. The responsivity factors and other LED characteristics are listed in Table 2. An important role play images of samples of the Murchison meteorite. Several calibration quantities $\left(\mathbf{F}, \mathbf{V}_{i}\right)$ are normalized with respect to the average inside a small circle (6 pixels radius) centered at $(x, y)=(484,553)$, at the location of a pellet containing a Murchison sample inside the image frame. The responsivity factors $R_{i}$ were derived by imaging, for each of the LED colors, a calibrated $\mathrm{BaSO}_{4}$ plate at a distance of $720 \mathrm{~mm}$ in an orientation perpendicular to the camera aperture. The reflectance factor of the white plate was a constant $0.968 \pm 0.015$ over the entire wavelength range of our LEDs. We measured the flux integrated over the LED spectral band (in $\mathrm{W} \mathrm{m}^{2}$ ) using a calibrated photometer. We also measured the average image signal (in $\mathrm{DN} \mathrm{s}^{-1}$ ) at the exact position of the photometer aperture. The ratio of these numbers gives the responsivity factors in Table 2 .

The angular distribution of light is similar but not the same for the different LEDs (Fig. 19). If one constructs color images this leads to color gradients not inherent to the surface. However, this can be corrected for with help of color ratio images. This will work best if the scene in front of the camera corresponds to the "nominal landing geometry", i.e. a flat surface as seen from the camera with MASCOT in the nominal landing position (bottom down). We simulated the nominal geometry in the lab with the $\mathrm{BaSO}_{4}$ plate. We acquired images of the plate with LED illumination for each of the four colors and cleaned them according to Eq. (1). The color ratio images $\mathbf{V}_{i}$ in Eq. (5) were then constructed as the ratio of the Blue, Red, and $I R$ images with respect to the Green image (22. 6). So $\mathbf{V}_{1}$ is Red/Green, $\mathbf{V}_{2}$ is the unity image, $\mathbf{V}_{3}$ is Red/Green, and $\mathbf{V}_{4}$ is IR/Green. Thus, images corrected with these ratio images will have an illumination pattern similar to that of the Green LEDs. The ratio images were subsequently smoothed and normalized to the average signal at the location of the Murchison pellet. Strictly speaking, they are only valid for surfaces with the nominal landing geometry, as other geometries may have different illumination patterns due to parallax.

We imaged the samples in all LED colors and processed them with the full calibration pipeline (Eqs. (1) and (5)). Figure 20 shows the result of an attempt to construct a "true" color image of the samples, displayed as a combination of the relative reflectance images in the Red, Green and Blue channel. The appearance of the samples is as if illuminated by white light. The reflectance spectrum of the small pellet will be measured independently. By comparison, the Murchison images will allow us to convert the radiance in Eq. (5) into relative reflectance of the surface in front of the camera. Deriving the absolute reflectance of objects in the FOV after landing on the asteroid is going to be very challenging, considering that the distance to those objects will be unknown. Part of the Hayabusa 2 commissioning was also a first health check of MASCOT and its payload. This was conducted on the 16th of December 2014, 13 days after launch. Part of this health check was a basic functional testing of MasCam. During this test, eight images were acquired, among them several LED-illuminated images, in order to evaluate the performance of the LED illumination unit (Fig. 21). 

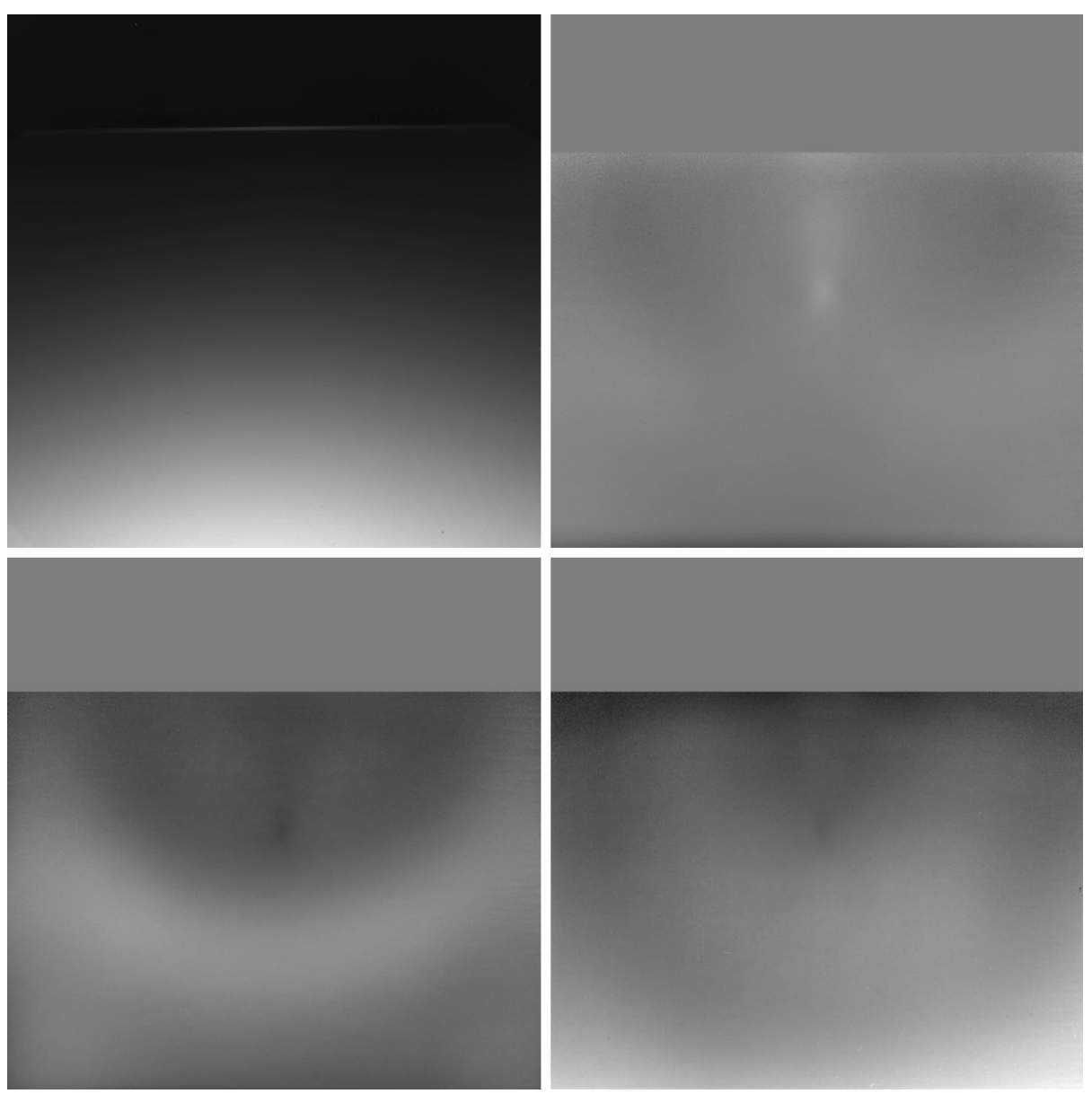

Fig. 19 LED ratio images $V_{i}$ as constructed from images of a $\mathrm{BaSO}_{4}$ plate in the nominal landing geometry. Top left: Green image of the illuminated plate. Top right: Blue over Green ratio image $\left(V_{1}\right)$. Bottom left: Red over Green ratio image $\left(V_{3}\right)$. Bottom right: IR over Green ratio image $\left(V_{4}\right)$. The brightness of the ratio images as displayed is such that 0.7 is black and 1.3 is white

\section{Operations}

MasCam must autonomously carry out its imaging sequences after separation from Hayabusa 2. In addition to that, the available downlink volume is very limited. To reduce the data volume a method to determine the image quality was implemented. One example for this is an algorithm that measures the amount of "dark" pixels and discards images with a certain percentage of these "dark" pixels. This is to ensure that e.g. descent images, which only show dark space, are not transmitted. Auto-exposure has also been implemented. In order to increase the reliability for optimal recording, the actual intelligence lies in the definition of acquisition sequences and not in the algorithms themselves. Table 3 lists and describes all MasCam algorithms which are used in various phases of the MASCOT Mission. 

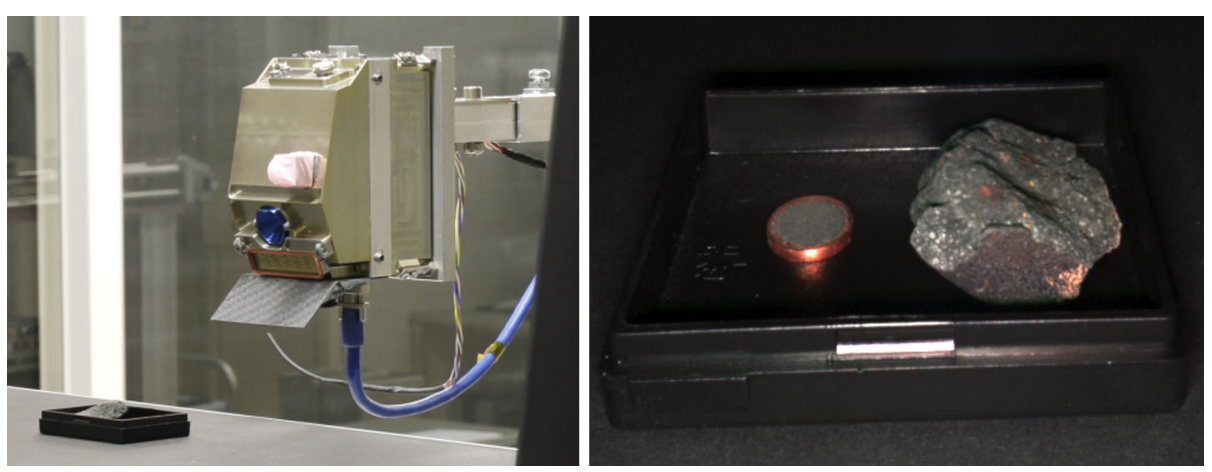

Fig. 20 Imaging Murchison meteorite samples (courtesy ONC team). A box with two meteorite samples was placed on a sheet of black paper. The pellet sample with the copper ring is used for calibration purposes. Left: A photo of the lab setup. Right: A calibrated MASCAM color image produced by combining Blue, Green, and Red LED images. The image brightness is scaled in such a way that the meteorite brightness is maximum but not saturated
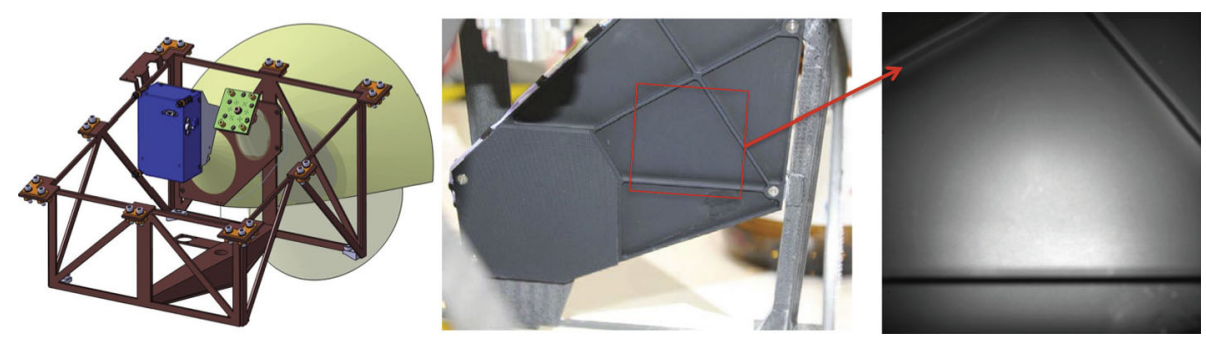

Fig. 21 (a) CAM view on the MASCOT calibration target. (b) MASCOT calibration target (flight model). (c) "First light" image (bias-corrected) of the MASCOT calibration target, acquired with the Red LED illumination, exposure time $=159.9 \mathrm{~ms}$, lossless compression). In flight configuration, the calibration target is accommodated slightly below the camera's focus range. Hence, the image is out of focus. The bright random pattern in the lower right corner of the image is a known production damage on the surface of the calibration target

All imaging operations are coded in time line sequences (Table 4) which are executed and controlled by events. One important event is to detect the transition period from day to night, in order to trigger the start of the imaging sequence using LED illumination.

\section{Ground Segment, Data Products \& Processing, Archiving}

The MasCam data will be received at the JAXA ground stations together with all other Mascot instrument data. The data will be transferred from JAXA to the MASCOT Control Center (MCC) located at the Microgravity User Support Center (MUSC) at DLR in Cologne. MUSC will sort the data for the different instruments and will combine them with the necessary housekeeping data. MasCam data will be converted to images in the VICAR (Video Image Communication And Retrieval) format (level-1 data product). Subsequently, the radiometric calibration (dark current and flat field correction) and the correction for geometric distortion will be applied, resulting in calibrated (level-2) products. Both level-1 and level-2 will be collected in a database at DLR Berlin, and converted to PDS-4 data. These 
Table 3 MasCam onboard algorithms

Histogram calculation

Histogram statistic

Image statistic

Auto exposure

High Dynamic Range Image (HDR)

Basic arithmetic operations

Compression
After the separation will go into MASCOT uncontrolled rotation. For reducing the data volume with the histogram calculation and the definition of a threshold is possible to sort out deep space images.

Calculated from a histogram of the mean, standard deviation, minimum and maximum values. This function is required for a health check of the camera if it is not possible to transmit pictures.

Calculated from an image the mean, standard deviation, minimum and maximum values.

After MASCOT tough the surface and stops bouncing, the commanded exposure time must be adapted to the illuminated surface and environment. By specification of acceptable image saturation, deviation of maximum and minimum exposure time, it is possible to automatically determine an optimal exposure time.

With this method it is possible to increase with three pictures the dynamic range. This method works similarly to the auto exposure but only with additional parameters which defines over and a under exposed image. These images are merged together after transferring to the earth into an HDR image.

Accumulation, subtraction, division, multiplication on one image with a factor or between on a set of images.

The MASCOT compression based on a wavelet transformation. Before compression the images are divided into $128 \times 128$ tiles to decrease the risk losing a whole image if one bye is lost during the transmission. The following compression ratios are implemented: lossless bit packing, 1:4, $1: 8,1: 16$

Table 4 MasCam imaging sequences

CAM_SEQ_SDL_Image sequence with a fix exposure time which operated during the separation, descent and landing/bouncing phase. The time offset between the images is flexible and not in a equidistant range so is possible to decide in which height over the surface the camera takes more images in a better resolution.

CAM_SEQ_HOP This image sequence has also a fix exposure time and will be operated during the hopping and reorientation of MASCOT.

CAM_SEQ_PHO This image photometry sequence needs different sun phase angles positions requires an auto expo-sure cycle before each image.

CAM_SEQ_LED LED light image sequence during night in the first night an auto exposure cycle is required for each LED wavelength. Between each LED image is possible to define a delay time to control the temperature. The Onboard computer for store all LED images the used exposure time. The LED sequence start and ends with a dark image.

CAM_SEQ_HDR Image sequence with different exposure times. Starts with auto exposure time calculation then the exposure time will be incremented and decrement by a user defined exposure time offset.

will then be transferred back to JAXA, who will stored them in the long-time archive and make them available to the public.

Acknowledgements The authors gratefully acknowledge the support of the MASCOT and Hayabusa 2 engineering and operations teams. Samples of the Murchison pellet were kindly provided by the Hayabusa 2 ONC team for the purpose of cross-calibration. 


\section{References}

M. Abe, Y. Takagi, K. Kitazato, S. Abe, T. Hiroi, F. Vilas, B.E. Clark, P.A. Abell, S.M. Lederer, K.S. Jarvis, T. Nimura, Y. Ueda, A. Fujiwara, Near-infrared spectral results of asteroid Itokawa from the Hayabusa spacecraft. Science 312, 1334-1338 (2006). doi:10.1126/science.1125718

J. Bell, J. Mitton (eds.), Asteroid Rendezvous-NEAR Shoemaker's Adventures at Eros (Cambridge Univ. Press, Cambridge, 2002). ISBN 0-521-81360-3

J.P. Bibring, et al., Space Sci. Rev. (2016, this issue)

R.P. Binzel, A.W. Harris, S.J. Bus, T.H. Burbine, Spectral properties of near-Earth objects: Palomar and IRTF results for 48 objects including spacecraft targets (9969) Braille and (10302) 1989 ML. Icarus 151, 139-149 (2001)

R.P. Binzel, D.F. Lupishko, M. Di Martino, R.J. Whiteley, G.J. Hahn, in Asteroids III, ed. by W.F. Bottke et al. (Univ. of Arizona Press, Tucson, 2002), p. 255

S.J. Bus, R.P. Binzel, Phase II of the small main-belt asteroid spectroscopic survey. A feature-based taxonomy. Icarus 158, 146-177 (2002a)

S.J. Bus, R.P. Binzel, Phase II of the small main-belt asteroid spectroscopic survey. The observations. Icarus 158, 106-145 (2002b)

H. Campins, J.P. Emery, M. Kelley, Y. Fernández, J. Licandro, M. Delbó, A. Barucci, E. Dotto, Spitzer observations of spacecraft target 162173 (1999 JU3). Astron. Astrophys. 503, L17-L20 (2009). doi:10.1051/0004-6361/200912374

H. Campins, J. de León, A. Morbidelli, J. Licandro, The origin of asteroid 162173 (1999 JU3). Astron. J. 146, 26 (2013). doi:10.1088/0004-6256/146/2/26

A. Fujiwara, J. Kawaguchi, D.K. Yeomans, M. Abe, T. Mukai, T. Okada, J. Saito, H. Yano, M. Yoshikawa, D.J. Scheeres, O. Barnouin-Jha, A.F. Cheng, H. Demura, R.W. Gaskell, N. Hirata, H. Ikeda, T. Kominato, H. Miyamoto, A.M. Nakamura, R. Nakamura, S. Sasaki, K. Uesugi, The rubble-pile asteroid Itokawa as observed by Hayabusa. Science 312, 1330-1334 (2006). doi:10.1126/science.1125841

M. Grott, J. Knollenberg, B. Borgs, F. Hänschke, E. Kessler, J. Helbert, A. Maturilli, N. Müller, The MASCOT radiometer MARA for the Hayabusa 2 mission. Space Sci. Rev. (2016, this issue)

J.T. Grundmann, U. Auster, V. Baturkin, A. Bellion, J.-P. Bibring, J. Biele, R. Boden, O. Bompis, B. Borgs, P. Bousquet, E. Canalias, L. Celotti, C. Cenac-Morthe, F. Cordero, M. Deleuze, C. Evesque, R. Findlay, S. Fredon, K.-H. Glaßmeier, D. Granena, C.D. Grimm, M. Grott, V. Hamm, J. Hendrikse, D. Herčík, T.-M. Ho, R. Jaumann, C. Krause, R. Kroth, E. Ksenik, C. Lange, M. Lange, O. Mierheim, T. Okada, J. Reill, K. Sasaki, N. Schmitz, H.-J. Sedlmayr, M. Talapina, S. Tangruamsub, N. Termtanasombat, S. Ulamec, E. Wejmo, M. Wrasmann, T. Yoshimitsu, C. Ziach (the MASCOT Team), Mobile asteroid surface scout (MASCOT) - design, development and delivery of a small lander aboard Hayabusa 2, in 4th IAA Planetary Defense Conference-PDC, AA-PDC-15-P-64 (2015)

S. Hasegawa, T.G. Müller, K. Kawakami, T. Kasuga, T. Wada, Y. Ita, N. Takato, H. Terada, T. Fujiyoshi, M. Abe, Albedo, size, and surface characteristics of Hayabusa 2 sample-return target 1621731999 JU3 from AKARI and Subaru observations. Publ. Astron. Soc. Jpn. 60, S399-S405 (2008)

D. Hercik, H.-U. Auster, J. Blum, K.-H. Fornacon, M. Fujimoto, K. Gebauer, C. Güttler, O. Hillenmaier, A. Hördt, E. Liebert, A. Matsuoka, R. Nomura, I. Richter, B. Stoll, B. Weiss, K.-H. Glassmeier, MasMag: The MASCOT magnetometer experiment. Space Sci. Rev. (2016). doi:10.1007/s11214-016-0236-5

T.-M. Ho, V. Baturkin, R. Findlay, C. Grimm, J.T. Grundmann, C. Hobbie, E. Ksenik, C. Lange, K. Sasaki, M. Schlotterer, M. Talapina, N. Termtanasombat, L. Witte, M. Wrasmann, G. Wübbels, J. Rößler, C. Ziach, J. Biele, C. Krause, S. Ulamec, M. Lange, O. Mierheim, R. Lichtenheldt, M. Meier, J. Reill, H.J. Sedlmayr, P. Bousquet, A. Bellion, O. Bompis, C. Cenac-Morthe, M. Deleuze, S. Fredon, E. Jurado, E. Canalias, R. Jaumann, J.-P. Bibring, K.H. Glassmeier, M. Grott, L. Celotti, F. Cordero, J. Hendrikse, MASCOT_The Mobile Asteroid Surface Scout onboard the Hayabusa2 Mission. Space Sci. Rev. (2016, this issue). doi:10.1007/s11214-016-0251-6

R. Jaumann, J.P. Bibring, K.H. Glassmeier, M. Grott, T.-M. Ho, S. Ulamec, N. Schmitz, H.-U. Auster, J. Biele, H. Kuninaka, T. Okada, M. Yoshikawa, S. Watanabe, M. Fujimoto, T. Spohn, Mobile asteroid surface scout (MASCOT) for the Hayabusa 2 mission to 1999 JU3: the scientific approach, in 44th Lunar and Planetary Science Conf., abstract \#1500 (2013)

M.-J. Kim, Y.-J. Choi, H.-K. Moon, H.-K.M. Ishiguro, S. Mottola, M. Kaplan, D. Kuroda, D.S. Warjurkar, J. Takahashi, Y.-I. Byun, Optical observations of NEA 162173 (1999 JU3) during the 2011-2012 apparition. Astron. Astrophys. 550, L11 (2013). ISSN 0004-6361. doi:10.1051/0004-6361/201220673

D. Lazzaro, M.A. Barucci, D. Perna, F.L. Jasmim, M. Yoshikawa, J.M.F. Carvano, Rotational spectra of (162173) $1999 \mathrm{JU} 3$, the target of the Hayabusa 2 mission. Astron. Astrophys. 549, L2 (2013). doi:10.1051/0004-6361/201220629

S. Mottola, G. Arnold, H.-G. Grothues, R. Jaumann, H. Michaelis, G. Neukum, J.P. Bibring, The ROLIS experiment on the Rosetta lander. Space Sci. Rev. 128, 241-255 (2007). doi:10.1007/s11214-006-9004-2 
T.G. Müller, J. Ďurech, S. Hasegawa, M. Abe, K. Kawakami, T. Kasuga, D. Kinoshita, D. Kuroda, S. Urakawa, S. Okumura, Y. Sarugaku, S. Miyasaka, Y. Takagi, P.R. Weissman, Y.-J. Choi, S. Larson, K. Yanagisawa, S. Nagayama, Thermo-physical properties of 162173 (1999 JU3), a potential flyby and rendezvous target for interplanetary missions. Astron. Astrophys. 525, A145 (2011). doi:10.1051/0004-6361/201015599

N. Schmitz, A. Koncz, R. Jaumann, H. Hoffmann, D. Jobs, J. Kachlicki, H. Michaelis, S. Mottola, B. Pforte, S. Schroeder, R. Terzer, F. Trauthan, M. Tschentscher, S. Weisse, T.-M. Ho, J. Biele, S. Ulamec, B. Broll, A. Kruselburger, L. Perez-Prieto, The MASCOT camera on Hayabusa 2's asteroid lander MASCOT (mobile asteroid surface scout), in 46th Lunar and Planetary Science Conf. (2015), abstract \#1799

J. Saito, H. Miyamoto, R. Nakamura, M. Ishiguro, T. Michikami, A.M. Nakamura, H. Demura, S. Sasaki, N. Hirata, C. Honda, A. Yamamoto, Y. Yokota, T. Fuse, F. Yoshida, D.J. Tholen, R.W. Gaskell, T. Hashimoto, T. Kubota, Y. Higuchi, T. Nakamura, P. Smith, K. Hiraoka, T. Honda, S. Kobayashi, M. Furuya, N. Matsumoto, E. Nemoto, A. Yukishita, K. Kitazato, B. Dermawan, A. Sogame, J. Terazono, C. Shinohara, H. Akiyama, Detailed images of asteroid 25143 Itokawa from Hayabusa. Science 312, 1341-1344 (2006). doi:10.1126/science.1125722

S. Sugita, T. Morota, S. Kameda, R. Honda, C. Honda (Hayabusa 2 ONC Science Team), Science observations strategy for HAYABUSA 2 optical navigation cameras (ONC), in 44th Lunar and Planetary Science Conf. (2013), abstract \#3026 (http://www.lpi.usra.edu/meetings/lpsc2013/eposter/3026.pdf)

S. Tachibana, M. Abe, M. Arakawa, M. Fujimoto, Y. Iijima, M. Ishiguro, K. Kitazato, N. Kobayashi, N. Namiki, T. Okada, R. Okazaki, H. Sawada, S. Sugita, Y. Takano, S. Tanaka, S. Watanabe, M. Yoshikawa, H. Kuninaka, Hayabusa2: Scientific importance of samples returned from C-type near-Earth asteroid (162173) 1999 JU3. Geochem. J. 48(6), 571-587 (2014). doi:10.2343/geochemj.2.0350

D. Tholen, M.A. Barucci, in Asteroids II, ed. by R.P. Binzel, T. Gehrels, M.S. Matthews (Univ. of Arizona Press, Tuscon, 1989), p. 298

S. Ulamec, J. Biele, P.-W. Bousquet, P. Gaudon, K. Geurts, T.-M. Ho, C. Krause, C. Lange, R. Willnecker, L. Witte, Landing on small bodies: from the Rosetta lander to MASCOT and beyond. Acta Astronaut. 93, 460-466 (2014)

F. Vilas, Special analysis of Hayabusa 2 near Earth asteroid targets 1621731999 JU3 and 2001 QC3. Astron. J. 135, 1101-1105 (2008). doi:10.1088/0004-6256/135/4/1101 